Research Articles: Systems/Circuits

\title{
Human NREM Sleep Promotes Brain-Wide Vasomotor and Respiratory Pulsations
}

https://doi.org/10.1523/JNEUROSCI.0934-21.2022

Cite as: J. Neurosci 2022; 10.1523/JNEUROSCI.0934-21.2022

Received: 1 May 2021

Revised: 10 January 2022

Accepted: 11 January 2022

This Early Release article has been peer-reviewed and accepted, but has not been through the composition and copyediting processes. The final version may differ slightly in style or formatting and will contain links to any extended data.

Alerts: Sign up at www.jneurosci.org/alerts to receive customized email alerts when the fully formatted version of this article is published.

Copyright (c) 2022 Helakari et al.

This is an open-access article distributed under the terms of the Creative Commons Attribution 4.0 International license, which permits unrestricted use, distribution and reproduction in any medium provided that the original work is properly attributed. 


\section{Human NREM sleep promotes brain-wide vasomotor and respiratory pulsations}

2 Abbreviated title: NREM sleep promotes physiological brain pulsations

3 Helakari $\mathbf{H}^{1,2,3}$, Korhonen $\mathbf{V}^{1,2,3}$, Holst $\mathrm{SC}^{4}$, Piispala $\mathbf{J}^{2,3,5}$, Kallio $\mathbf{M}^{2,3,5}$, Väyrynen $\mathbf{T}^{1,2,3}$, Huotari $\mathbf{N}^{1,2,3}$,

4 Raitamaa $\mathbf{L}^{1,2,3}$, Tuunanen $\mathbf{J}^{1,2,3}$, Kananen $\mathbf{J}^{1,2,3}$, Järvelä $\mathbf{M}^{1,2,3}$, Tuovinen $T^{1,2,3}$, Raatikainen $\mathbf{V}^{1,2,3}$, Borchardt

$5 \quad \mathbf{V}^{1,2,3}$, Kinnunen $\mathbf{H}^{6}$, Nedergaard $\mathbf{M}^{7,8}$ and Kiviniemi $\mathbf{V}^{1,2,3}$

6 Affiliations

7 1* $^{*}$ Oulu Functional Neuroimaging (OFNI), Department of Diagnostic Radiology, Oulu University Hospital, 90220

8 Oulu, Finland

92 Medical Imaging, Physics and Technology (MIPT), Faculty of Medicine, University of Oulu, 90220 Oulu,

10 Finland

$11 \quad{ }^{3}$ Medical Research Center (MRC), 90220 Oulu, Finland

$12 \quad{ }^{4}$ Neurobiology Research Unit, University of Copenhagen, 2200 Copenhagen, Denmark

$13{ }^{5}$ Clinical Neurophysiology, Oulu University Hospital, 90220 Oulu, Finland

$14 \quad{ }^{6}$ Oura Health Ltd.'s, 90590 Oulu, Finland

$15{ }^{7}$ Center of Translational Neuromedicine, University of Copenhagen, 2200 Copenhagen, Denmark

$16 \quad{ }^{8}$ Center of Translational Neuromedicine, University of Rochester, 14642 Rochester NY, US

$17{ }^{*}$ The work was performed in Oulu University Hospital

Corresponding Author

19 Vesa Kiviniemi, Prof, MD

20 vesa.kiviniemi@oulu.fi

21 Department of Diagnostic Radiology, Medical Research Center (MRC), Oulu University Hospital, Kajaanintie 50,

2290220 , Oulu

23 Heta Helakari, MSc

24 heta.helakari@oulu.fi 
Department of Diagnostic Radiology, Medical Research Center (MRC), Oulu University Hospital, Kajaanintie 50, 90220, Oulu

Number of figures: 6

Number of tables: 2

Number of words for abstract: 264

Number of words for introduction: 752

Number of words for discussion: 1594

\section{Limitations and Conclusions requested by both reviewers:}

Discussion (if limitations included): 1837

Number of words for Limitations: 242

Number of words for Conclusions: 109

\section{Conflict of interest}

Financial Disclosure: none

Non-financial Disclosure: none

Oura Health Ltd.'s provided the surveillance smart rings for the study, but did not participate in the analysis. The manuscript in question is published as a preprint in bioRxiv, but we guarantee that it does not infringe any subsequent copyright or license agreement.

\section{Acknowledgements}

This work was supported by Uniogs/MRC Oulu DP-grant (HH, JK), Pohjois-Suomen Terveydenhuollon tukisäätiö (HH, VKo), JAES-Foundation (VKi), Academy of Finland and Aivosäätiö TERVA grant 314497 (VKi), Academy of Finland Grant 275342 (VKi), The SalWe Research Program for Mind and Body (Tekes—the Finnish Funding Agency for Technology and Innovation, Grant No. 1104/10) (VKi), The Finnish Medical Foundation (VKi, JK, TT), Finnish Neurological Foundation (Vki), KEVO grants from Oulu University hospital (VKi), Orion Research Foundation sr (JK, TT), Medical Research Center (MRC) -Oulu (JK, VR), Maire Taponen Foundation sr (JK), Finnish Brain Foundation sr (JK, VKi), Instrumentarium Science Foundation sr (JK), The University of Oulu Scholarship Foundation (JK, VR). 
51 We would like to thank all study subjects for their participation in the study. We also thank Tuomas Konttajärvi for

52 assistance in measurements and preprocessing of EEG data, Jani Häkli, Annastiina Kivipää, Tarja Holtinkoski,

53 Aleksi Rasila, Taneli Hautaniemi, Miia Lampinen and others who assisted in measurements or otherwise

54 contributed. We are grateful for devices and data provided by Oura. We wish to acknowledge Jussi Kantola for data

55 management and reconstruction of MREG data, the CSC - IT Center for Science Ltd., Finland for generous

56 computational resources, Prof. Paul Cumming of Bern University Hospital for comments on the manuscript, and

57 Dan Xue for assistance with the figures.

58

59

60 
61 Abstract

62 The physiological underpinnings of the necessity of sleep remains uncertain. Recent evidence suggests that sleep increases the convection of cerebrospinal fluid (CSF) and promotes the export of interstitial solutes, thus providing a framework to explain why all vertebrate species require sleep. Cardiovascular, respiratory, and vasomotor brain pulsations have each been shown to drive CSF flow along perivascular spaces, yet it is unknown how such pulsations may change during sleep in humans. To investigate these pulsation phenomena in relation to sleep, we simultaneously recorded fast fMRI, magnetic resonance encephalography (MREG) and electroencephalography (EEG) signals in a group of healthy volunteers. We quantified sleep-related changes in the signal frequency distributions by spectral entropy analysis and calculated the strength of the physiological (vasomotor, respiratory, and cardiac) brain pulsations by power sum analysis in 15 subjects (aged $26.5 \pm 4.2$ years, 6 females). Finally, we identified spatial similarities between EEG slow oscillation (0.2-2 Hz) power and MREG pulsations. Compared to wakefulness, non-rapid eye movement (NREM) sleep was characterized by reduced spectral entropy and increased brain pulsation intensity. These effects were most pronounced in posterior brain areas for very low frequency $(\leq 0.1$ $\mathrm{Hz}$ ) vasomotor pulsations but were also evident brain-wide for respiratory pulsations, and to a lesser extent for cardiac brain pulsations. There was increased EEG slow oscillation power in brain regions spatially overlapping with those showing sleep-related MREG pulsation changes. We suggest that reduced spectral entropy and enhanced pulsation intensity are characteristic of NREM sleep; together with our findings of increased power of slow oscillation, present results support the proposition that sleep promotes fluid transport in human brain.

\section{Statement of Significance}

We report that the spectral power of physiological brain pulsation mechanisms driven by vasomotor, respiration and cardiac rhythms in human brain increase during sleep, extending previous observations of their association with glymphatic brain clearance during sleep in rodents. The magnitudes of increased pulsations follow the rank order vasomotor > respiratory > cardiac pulsations, with correspondingly declining spatial extents. Spectral entropy, previously known as vigilance and as an anesthesia metric, decreased during NREM sleep compared with the awake state in very low and respiratory frequencies, indicating reduced signal complexity. An EEG slow oscillation power increase occurring in the early sleep phase (NREM 1-2) spatially overlapped with pulsation changes, indicating reciprocal mechanisms between those measures. 


\section{Introduction}

A notable feature of the mammalian brain is that it consists of almost $80 \%$ water (Tait et al. 2008), with a near absence of connective tissue. Also, the brain floats in cerebrospinal fluid (CSF) within the closed cavity of the skull. These physical characteristics enable brain-wide pulsations that drive fast fluid movement within the neuropil. In the early $20^{\text {th }}$ century Hans Berger described three forms of brain pressure pulsations: "eine pulsatorische, eine respiratorische und vasomotorische Bewenung” (Berger 1901); his studies of pulsatile brain activity lead the invention of electroencephalography (EEG).

Nearly a century later, the same triad of cardiovascular, respiratory and slow, spontaneous vasomotor waves driven by arteries can be non-invasively imaged throughout the brain by magnetic resonance encephalography (MREG), which is a fast variant of functional magnetic resonance imaging (fMRI) (Kiviniemi et al. 2016) designed to maximize temporal accuracy while maintaining adequate image quality. Besides MREG, several fMRI imaging techniques have shown distinct cardiac, respiratory (Dreha-Kulaczewski et al. 2015; Klose et al. 2000; Matsumae et al. 2019; Posse et al. 2013; Sloots et al. 2020; Yamada et al. 2013) and very low frequency (<0.1 Hz) (Fultz et al. 2019) pulsations in the living human brain. The pulsations must bear some relation with the normal resting heart rate, which ranges between 1-1.67 Hz in humans (Mason et al. 2007) and respiratory rate, which is within or close to range $0.16-0.33 \mathrm{~Hz}$ (Russo et al. 2017); both of these rates decrease during non-rapid eye movement (NREM sleep) (Penzel et al. 2003). Compared to other techniques, MREG enables robust detection and separation of physiological brain pulsations in the whole brain due to the greater spectral resolution in the range of $0-5 \mathrm{~Hz}$, which is obtained without signal aliasing (Huotari et al. 2019; Raitamaa et al. 2018; Tuovinen et al. 2020).

Sleep is known to elicit electrohydrodynamic changes in brain tissue (Ding et al. 2016) that are driven by the physiological pulsations (Mestre et al. 2018). Also, prior reports in rodents showed that natural sleep and certain anesthetic regimens can induce a sharp increase in the influx of CSF to brain tissue, which further announce an increase in slow wave electrophysiological activity (Hablitz et al. 2019; Xie et al. 2013). A recent study in mice associated $0.1 \mathrm{~Hz}$ vasomotor wave activity with increased paravascular solute transport (van Veluw et al. 2020), thus serving as an additional driver of fluid convection supplementing the previously presented cardiovascular brain pulsatility (Iliff et al. 2013; Mestre et al. 2018). In humans, NREM sleep and drowsiness are associated with high amplitude of very low frequency fluctuations of blood-oxygen-level-dependent (BOLD) signal (Chang et al. 
2016; Fukunaga et al. 2006; Horovitz et al. 2008; Wong et al. 2016), which are suggested to arise from vasomotion (Biswal et al. 1995; Kiviniemi et al. 2000; Rayshubskiy et al. 2014; Silvani et al. 2004; Wang et al. 2008). Furthermore, simultaneous EEG-fMRI study in humans linked increased delta wave amplitude during sleep with decreased cerebral blood volume followed by increased flow of CSF into the fourth ventricle (Fultz et al. 2019). Also, the previous human neuroimaging literature suggests that respiratory pulsation is the main pulsatory driver of CSF flow and intracerebral venous blood flow (Dreha-Kulaczewski et al. 2015; Matsumae et al. 2019; Vinje et al. 2019). Based on these various findings, we concluded that brain pulsations may affect the influx of CSF in a manner related to the state of consciousness and in association with slow wave electrophysiological activity.

Based on this scenario, we used whole brain MREG with synchronous EEG sleep monitoring during wakefulness and sleep to test the hypotheses that MREG pulsations change during sleep, and that the pulsations occur in association with EEG slow oscillations. By inducing a T2* MRI signal, MREG allows us to study brain fluid dynamics originating not only from blood oxygenation (i.e. susceptibility related BOLD effect), but also the dynamics arising from the CSF space and interstitial fluid changes. The short repetition time (TR $100 \mathrm{ms)} \mathrm{and} \mathrm{the}$ used read pulse flip angle (FA $5^{\circ}$ ), both increase inflow effects and sensitize detection of the steady state precession alterations induced in the brain MRI data by relatively sudden cardiorespiratory impulses. We studied spectral entropy and the power sum of the MREG signal to assess sleep-related pulsation changes and demonstrated their relation to EEG slow oscillations $(0.02-2 \mathrm{~Hz})$ with spatial co-localization. Overall, we found significant changes in vasomotor, respiratory, and cardiac pulsations during NREM sleep compared to awake, mostly in brain areas overlapping with those showing EEG slow oscillation changes.

\section{Materials and Methods}

\section{Subjects}

Twenty-five subjects (aged $28.0 \pm 5.9$ years, 11 females) participated in the study, which was approved by the Regional Ethics Committee of the Northern Ostrobothnia Hospital District. Written informed consent was obtained from all participants, according to requirements of the Declaration of Helsinki. Subjects were recruited from among university students by advertisement. Inclusion criteria for the study were sent via e-mail to all subjects, who were then interviewed to screen out cases meeting exclusion criteria. All subjects were healthy as assessed by the 
interview and met the following inclusion criteria: no continuous medication, no neurological or cardio-respiratory diseases, non-smokers and no pregnancy. We did not undertake screening for sleep apnea or other sleep disorders. The subjects were instructed to not consume caffeine four hours preceding the Awake scan session and eight hours preceding the Sleep scan session. Subjects were further instructed to abstain from alcohol during the night of sleep deprivation. Sample sizes of 15 (aged $26.5 \pm 4.2$ years, 6 females) and 12 (26.2 \pm 4.3 , 5 females) were used for the main analysis (Figure 1).

\section{Insert Figure 1 about here.}

\section{Data collection}

Subjects were scanned after a normal night's sleep $(7.8 \pm 1.3$ hours sleep the previous night) in the afternoon starting at 4-6 PM (and three days later after a monitored interval of wakefulness lasting $24 \pm 1.3$ hours) in the early morning starting at 6-8 AM (Figure 2a). We had sleep deprived the subjects aiming to maximize the likelihood of the subjects entering a deeper and more consolidated sleep state during the next day's MR imaging session (Horovitz et al. 2009; Kaufmann et al. 2006). At home, the sleep deprivation was verified by a smart ring (see details below). Subjects were instructed to wear the ring for at least 24 h preceding the Awake and Sleep scan sessions. The smart ring records upper limb motion (3-D accelerometer, $50 \mathrm{~Hz}$ ), photoplethysmogram (250 Hz) and skin temperature (1/min). Validation studies for polysomnography showed $96 \%$ sensitivity for detecting sleep, $48 \%$ specificity for detecting awake state, and medical-grade actigraphy ( $r=0.86$; correlation in total sleep time), thus confirming that the ring signals adequately separate awake from sleep states (de Zambotti et al. 2017; Mehrabadi et al. 2020). Smart ring data were available for 14 of 15 scanning subjects.

All subjects were scanned in Oulu (Finland) using a Siemens MAGNETOM Skyra 3T (Siemens Healthineers AG, Erlangen, Germany) scanner with a 32-channel head coil. The subjects were scanned with the MREG fast fMRI sequence, in synchrony with a previously described multimodal scanning setup (Korhonen et al. 2014). MREG is a single-shot sequence undersampling k-space with an in/out stack-of-spiral trajectories in three dimensions (Assländer et al. 2013; Lee et al. 2013; Zahneisen et al. 2012). The following parameters were used for MREG: repetition time $(\mathrm{TR}=100 \mathrm{~ms})$, echo time $(\mathrm{TE}=36 \mathrm{~ms})$, and flip angle $\left(\mathrm{FA}=5^{\circ}\right)$, field of view $(\mathrm{FOV}=192 \mathrm{~mm})$ and 3 mm cubic voxels. Parameters for three-dimensional structural T1 MPRAGE were TR $=1900 \mathrm{~ms}$, TE $=2.49$ ms, $\mathrm{FA}=9^{\circ}, \mathrm{FOV}=240 \mathrm{~mm}$, and slice thickness $0.9 \mathrm{~mm}$. MREG data were reconstructed using L2-Tikhonov 
regularization with lambda 0.1, where the latter regularization parameter was determined by the L-curve method with a MATLAB recon-tool provided by the sequence developers (Hugger et al. 2011).

Ear plugs were used to reduce perception of scanner noise, while head cushions restricted movement. During the Awake scan session, two MREG scans with simultaneous EEG and cardiorespiratory signals were recorded: I) tenminute resting state, Eyes open, Awake scan $\left(\mathrm{A}_{1-2}\right)$ fixating on a cross on the screen, II) five-minute resting state, Eyes closed (EC) (Figure 2a) with ambient light. Three days later during the Sleep scan session, one or two MREG sequences were recorded: $\mathrm{I})$ the first ten-minute Sleep scan $\left(\mathrm{S}_{1-2}\right)$, II) the second ten-minute Sleep scan $\left(\mathrm{S}_{3-4}\right)$, without ambient light during which the subjects were allowed to fall asleep ad libitum. Subjects were advised to contact the staff if they were not feeling somnolent after the first scan, which resulted in termination of the session for two of 15 subjects. An anatomical MR scan was performed at the end of both sessions.

When the subject was asleep when the scanning ended, lights were turned on and the subject was slowly woken up while remaining in the scanner. Due to individual differences in awake vigilance and ability to sleep in the MRI scanner, the ten-minute scans were divided into five-minute segments $\left(A_{1}, A_{2}, S_{1}, S_{2}, S_{3}\right.$ and $\left.S_{4}\right)$ to separate the awake and sleep states more effectively (Figure 2a). We used five-minute segments because the accuracy of spectral analysis of very low frequency pulsations increases as a function of the data recording length. For further analysis, we selected the segment (from $\mathrm{S}_{1}-\mathrm{S}_{4}$ ) with the highest sleep content, such that the Sleep data used for comparisons contained $91 \pm 11 \% \mathrm{~N} 1$ or $\mathrm{N} 2$ sleep $\left(\mathrm{A}_{1}\right.$ versus $\left.\mathrm{S}_{\mathrm{n}}\right)$.

EEG (0.01 Hz high-pass filtering) was recorded using the Electrical Geodesics (EGI, Magstim Company Ltd, Whitland, UK) MR-compatible GES 400 system, with a 256-channel high density net. Electrode impedances were $<50 \mathrm{k} \Omega$ and the sampling rate $1 \mathrm{kHz}$ (data from six subjects was sampled with $250 \mathrm{~Hz}$ by mistake, from which two (one $\mathrm{A}_{1-2}$ and one EC) EEG data sets were not usable for sleep scoring). Prior to scanning, signal quality was tested outside the scanner room by recording 30 second epochs of EEG with eyes open and eyes closed and by visually checking all the channels. Respiratory belt and fingertip peripheral $\mathrm{SpO}_{2}$ and anesthesia monitor data (ECG, fingertip peripheral $\mathrm{SpO}_{2}$ and end-tidal carbon dioxide $\left(\mathrm{EtCO}_{2}\right)$, Datex-Ohmeda $\mathrm{S} / 5$ Collect software) were measured in synchrony with the EEG, as described previously (Korhonen et al. 2014). 
Prior to the imaging, the subjects performed the reaction time and paired-associate learning tests from CANTAB, Cambridge Neuropsychological Test Automated Battery, Cambridge, UK (CANTAB ${ }^{\circledR}$ 2019) on a tablet computer. The CANTAB test is a widely accepted standard for measuring cognitive state and vigilance level.

\section{Preprocessing and analysis of MREG data}

After reconstruction, MREG data were preprocessed and analyzed using FSL (5.09 BET software (Jenkinson et al. 2012; Smith 2002)), AFNI (Analysis of Functional NeuroImages, v2 (Cox 1996)) and MATLAB (R2019). The brain was extracted from structural 3D MPRAGE volumes using neck clean-up and bias field correction options (Smith 2002). The functional data preprocessing was performed using the FSL pipeline. Data were high-pass filtered with a cut-off frequency of $0.008 \mathrm{~Hz}$, and framewise head motion was then performed with FSL 5.08 MCFLIRT software (Jenkinson et al. 2002). MCFLIRT relative mean displacement values (mm) of scan segments $\left(A_{1}\right.$ versus EC, $S_{1}, S_{2}, S_{3}$ and $\left.S_{4}\right)$, Awake versus Sleep analysis $\left(A_{1}\right.$ versus $\left.S_{n}\right)$ did not differ significantly (paired ttest, $\mathrm{p}_{\text {all }}>0.11$ ). Spatial smoothing was performed with $f$ slmaths using a $5 \mathrm{~mm}$ FWHM Gaussian kernel. The highest spikes in the MREG data time series were removed using the 3dDespike function in AFNI. Data were then registered into the Montreal Neurological Institute (MNI152) space at 3-mm resolution for comparable analysis between subjects. One hundred time points (10 seconds each) were excluded from the beginning of both five and ten-minute data segments so as to reach steady state signal saturation. Accordingly, from the ten-minute data, the final 100 time points were extracted to get a time series of the same length. Finally, we generated data sets for 2861 time points $\left(\mathrm{A}_{1}-\mathrm{S}_{4}\right)$, which corresponds to approximately five minutes.

\section{Preprocessing and analysis of EEG data}

We employed standard EEG clean-up strategies and EEG sleep state scoring for datasets of the 15 subjects in whom we had simultaneously measured EEG and MREG. Due to instances of noisy EEG signals that were unsuitable for sleep scoring in three subjects, the final sample size in most of the MREG analysis was 12 (paired ttest, $A_{1}$ versus $S_{n}$ ). In EEG slow oscillation analysis, sample size was ten versus 12, because of 2/12 had no usable awake EEG. Data of all 15 subjects were used for calculating the accuracy of spectral entropy sleep detection (see next section).

EEG recordings were preprocessed using the Brain Vision Analyzer (Version 2.1; Brain Products) after file format conversion via BESA Research (Version 7.0). Gradient artifacts due to static and dynamic magnetic fields present 
during the MRI data acquisition and ballistocardiographic (BCG) artifacts were corrected using the average artifact subtraction method (Allen et al. 1998; Allen et al. 2000). The absence of gradient of BCG artifacts was verified by visual inspection. After preprocessing, EEG data were visualized according to the 10-20 system instructions for sleep state scoring. Two experienced specialists in clinical neurophysiology, who were trained in EEG sleep scoring performed the scoring in 30 second epochs according to AASM guidelines for clinical sleep studies (American Academy of Sleep Medicine, 2017), and the final decisions on sleep states were obtained by consensus. EEG epochs were scored as awake, N1 (light sleep), N2 (intermediate sleep with sleep spindles and/or Kcomplexes), N3 (slow wave sleep) or REM (sleep with rapid eye movements).

For slow oscillation power spectral analysis, we removed linear trends to exclude stable conductivity changes at the electrode-skin interface, which could otherwise have affected the performance of various processing steps. We used fast independent component analysis (FastICA) in combination with principal component analysis (PCA) dimension reduction (150 components) to transform the datasets into independent components, where artificial ICs were identified and removed with the help of EEGLAB SASICA-tool after visual confirmation. To furthermore minimize non-linearities and enhance the performance of ICA, we used a spike detection algorithm to remove the most prominent artifactual signal excursions. To keep the recordings intact, we interpolated the trends for the gaps and used data from intact signal epochs to fill the gap in the manner similar to inpainting. Bad channels removed prior to ICA were afterwards reintroduced using spherical interpolation. Recordings were referenced to linked mastoid electrodes, which were located near to other electrodes but record less brain activity. Next, we generated five-minute segments $\left(A_{1}-S_{4}\right)$ corresponding with the analyzed MREG data. Power estimates were then calculated using short-time Fourier transform combined with a Hamming window of 30 seconds length and 50\% overlap. We calculated relative power to attenuate the subject specific variability $\mathrm{RP}_{\mathrm{sw}}=\mathrm{P}_{\mathrm{sw}} / \mathrm{P}_{\text {tot }}$. The time average was used to gain one $\mathrm{RP}$ estimate for each subject. As previous studies have linked increased CSF flow to slow wave changes (Fultz et al. 2019), we chose to study the slow wave activity (SWA, 0.5-4 Hz) and slow oscillations (0.2-2 Hz). Our direct current EEG allowed us to evaluate frequencies $<0.5$ $\mathrm{Hz}$. To follow the previous literature, we set $0.2 \mathrm{~Hz}$ as the lower limit for slow oscillations, as these are known to increase in amplitude during N2 sleep (Brancaccio et al. 2020). The upper limit was chosen to meet the standard requirement in scoring of slow wave sleep.

\section{MREG signal spectral entropy analysis}


Spectral entropy from EEG and EMG is known to measure depth of anesthesia (Viertiö-Oja et al. 2004) and sleep (Mahon et al. 2008), and to correlate with the level of consciousness. Given the ten Hz sampling rate of MREG data, we supposed that calculation of its spectral entropy might present an alternative to EEG spectral entropy analysis by showing similar reductions during sleep (Kumar et al. 2013; Mahon et al. 2008; Vakkuri et al. 2005). We calculated a single spectral entropy value for whole time series with MATLAB (R2019) using the pentropy function, which produced a spatial map. The method is based on treating the normalized Fast Fourier Transform (FFT) power spectrum as a probability distribution, which is then used to calculate the Shannon entropy (Shannon 1948). The spectral entropy value describes the irregularity or complexity of the signal, where a noiseless, regular, and totally predictable signal such as a perfect sine wave has an entropy value of zero, whereas complex signals with several prominent frequency bands have a higher spectral entropy value. Thus, spectral entropy tends to decrease when the power in one frequency range starts to dominate.

We tested the accuracy of spectral entropy to discriminate sleep from wakefulness. First, we created a model that included data from only the awake and deepest sleep conditions. As there were 30 fully awake five-minute segments, we chose for comparison the 30 segments with the deepest sleep state (88 $\pm 12 \% \mathrm{~N} 1$ or N2). We weighted the sleep states incrementally with increasing depth of sleep (awake=0, N1=1, N2=2, N3=3), multiplied the weighting values by the corresponding number of 30 second epochs, and calculated the sum to get an EEGweighted sleep score for each five-minute segment. Thus, a fully awake five-minute session corresponds to a minimum EEG weighted sleep score of zero and a five-minute segment of entirely N3 sleep corresponds to a maximum score of 30 . Data were included when sleep states and spectral entropy were both available $\left(A_{1}: n=12\right.$, $A_{2}: n=12, E C: n=13, S_{1}: n=12, S_{2}: n=12, S_{3}: n=10$ and $\left.S_{4}: n=10\right)$.

To test the fitness of spectral entropy to accurately identify sleep, we chose a region of interest (ROI) from visual cortex (spherical ROI of $5 \mathrm{~mm}$ radius, MNI x 12 y $-63 \mathrm{z}-6$ ) for further analysis, as the fMRI signal in this region robustly changes across the sleep-wake cycle (Chang et al. 2016; Fukunaga et al. 2006; Liu et al. 2018). We then compared AASM sleep scores and the ROI spectral entropy results to see whether spectral entropy changes occurred prior to sleep onset (Figure 3a-b). Next, we calculated the receiver operating curve (ROC) to determine the separability, sensitivity and specificity of the ROI spectral entropy to distinguish sleep from wakefulness.

We then performed a voxel-level analysis to map regions in which spectral entropy distinguished between Awake versus Sleep states ( $A_{1}$ versus $S_{n}$; $n=12$ for whom sleep state available). Next, we applied a bandpass filter with 
3dTproject for very low $(0.008-0.1 \mathrm{~Hz})$, respiratory $(0.11-0.44 \mathrm{~Hz})$, and cardiac $(0.52-1.6 \mathrm{~Hz})$ frequencies to enable a comparison of spectral entropy in relation to these distinct physiological phenomena. The very low range of 0.008-0.1 Hz was chosen to obtain maximum possible coverage of the low frequencies, without cross-talk from respiratory frequencies. Group mean ranges for respiratory and cardiac frequencies were verified from individual anesthesia and/or scanner physiological monitoring signals for FFT analysis. As previously shown, cardiorespiratory distributions in MREG correspond to the frequency ranges in physiological signals (Tuovinen et al. 2020). First, we defined the peak of the respiratory frequency and then identified, the starting and ending points of the respiratory power (lowest point around the peak) from the $\mathrm{EtCO}_{2}$ signals; if not available, we used data from the respiratory belt of each scan separately. All subjects had some data available $\left(A_{1}-S_{4}\right)$ but in $7 / 60$ of the datasets we could not make the definition. Cardiac frequency range was defined similarly from the fingertip peripheral $\mathrm{SpO}_{2}$ (8/60 missing, but at least one dataset was available for all subjects). Group mean ranges were chosen based on the lowest and highest values of the individual ranges, with no overlap of other frequency bands.

\section{Power spectral analysis of MREG signal}

Using power spectrum analysis, we studied differences between $A_{1}$ versus $S_{n}$, voxel-wise and for the global signal, which is known to shift depending on state of arousal (Chang et al. 2016; Liu et al. 2018; Wen and Liu 2016). Power spectral analysis is an effective tool to separate different sources of brain fluctuations according to different frequency ranges (Duff et al. 2008). Here, we separated three frequency bands as described in the preceding section. For each frequency range, we calculated a FFT power density map with the 3dPeriodogram function in AFNI for the $A_{1}$ and $S_{1-4}$ scans. The scanning segments together comprised 2861 time points, and FFT was conducted with 4096 bins such that we ended up with 2048 bins corresponding to 0-5 Hz (0.0024 Hz resolution). Very low frequency, respiratory, and cardiac verified FFT power frequency ranges were separated from the global and voxel-wise MREG periodograms using fslroi (after specifying the frequency ranges), and the summed power over each frequency range was calculated using 3dTstat.

\section{Correlation analysis between spectral entropy and power}

Using fslcc, spectral entropy difference maps were correlated with the corresponding sum of power maps to evaluate the extent of their spatial overlap. The three frequency band results of both methods were likewise correlated to reveal whether the different pulsations involved similar brain regions. 


\section{Statistical analysis}

We used FSL randomise with Threshold-Free Cluster Enhancement and family-wise error (false positive) control along with a two-sample paired t-test model ( $A_{1}$ versus $S_{n}$, df 11$)$ in the voxel-wise spectral entropy and the FFT power analyses. We calculated Pearson correlations for sleep state change and spectral entropy ROI in relation to scan segments (from $A_{1}$ to $S_{2}$ ).

The ROC curve was calculated to reveal the accuracy of spectral entropy to distinguish sleep and awake states, and the optimal sensitivity and specificity of the model was calculated (MATLAB R2019). For smart ring activity, CANTAB data, and head motion data, statistical differences were calculated by a two-sample paired t-test model using SPSS (IBM SPSS Statistics 26). In the EEG slow oscillation analysis, statistical testing for the difference in means was made with permutation testing by randomly shuffling the group labels 10,000 times. We transformed the differences into z-scores, from which statistically significant (two-tailed, $\mathrm{p}<0.05$ ) results can be obtained.

\section{Results}

We studied spectral entropy and power sum of physiological brain pulsations during awake and NREM sleep and then, investigated their relation to EEG slow oscillation power. We performed main analysis with sample size of 12 or 15 (Figure 1). Data from a representative subject demonstrates the dramatic effect of NREM sleep on the power intensity of the EEG (Figure 2 b-c) and MREG data in the three extracted pulsation frequency ranges (Figure 2 de). Respiratory and very low frequency power increased sharply and spectral entropy decreased in NREM sleep (the chosen five-minute segment here includes 80\% N2 and 20\% N1 sleep), especially when entropy was calculated in the visual cortex (Figure 2b-e).

\section{Insert Figure 2 about here.}

\section{N1 and N2 states occurred as majority in Sleep scans}

Sleep scoring was performed for 15 subjects across the Awake and Sleep scan sessions. Only a single epoch of sleep was detected for one individual in the Awake eyes open $\left(\mathrm{A}_{1-2}\right)$ scan sessions. Among the Eyes closed scans, $32 \%$ were scored as NREM sleep, most of which ( 88\%) were N1. Finally, the Sleep scan sessions $\left(\mathrm{S}_{1-4}\right)$ included 72-87\% N1 or N2 sleep (Table 1, Figure 2a). 
To investigate effects of NREM sleep on MREG detected brain pulsations, we selected individual five-minute segments that included the highest amount of sleep. For subject level analysis $(n=12)$, the average amount of NREM sleep in the Sleep scan session was $91 \pm 11 \%$ (N1: $33 \pm 28 \%$, N2: $57 \pm 32 \%$, N3:1 $\pm 3 \%$, awake: $8 \pm 1$ \%, unknown: $1 \pm 3 \%$, whereas the awake scan $\left(\mathrm{A}_{1}\right)$ consisted of wakefulness and only $1 \pm 3 \%$ N1 sleep (Table 1).

\section{Insert Table 1 about here.}

\section{Frequency ranges for pulsation analysis were determined from physiological $\mathrm{EtCO}_{2}$ and $\mathrm{SpO}_{2}$ signals}

Group mean ranges for respiratory and cardiac frequencies were obtained from individual physiological monitoring $\mathrm{EtCO}_{2}$ or respiratory belt and $\mathrm{SpO}_{2}$ signal data based on the minimum and maximum points of the highest peak. The peaking value was significantly lower for $\mathrm{EtCO}_{2}$ in $\mathrm{S}_{1}(\mathrm{p}=0.012)$ and $\mathrm{S}_{2}(\mathrm{p}=0.029)$ when compared to $\mathrm{EO}$ and for $\mathrm{SpO}_{2}$ in $\mathrm{EC}(\mathrm{p}=0.0009)$ and $\mathrm{S}_{2}(\mathrm{p}=0.001)$, all when compared to $\mathrm{EO}$.

\section{Insert Table 2 about here.}

\section{Smart ring corroborated sleep deprivation and CANTAB revealed slower reaction time after sleep deprivation}

Based on pilot data for the successful measurement of synchronous multimodal MREG and DC-EEG signal (Korhonen et al. 2014), we recruited healthy subjects for a sleep monitoring study, and requested them to use the ring for at least 24 hours preceding the Awake scan session likewise to verify sleep deprivation preceding the Sleep scan session. Based on the smart ring data, we observed only $9 \pm 19$ minutes of sleep before Sleep scan sessions, corroborating that our subjects were indeed sleep deprived at that time (14 of 15 subjects). Three/15 subjects had incomplete smart ring data during the night of sleep deprivation (16, 25 and 57 minutes) and nine/15 subjects had some missing data during the entire 24 hours before the scan. After sleep deprivation, the subjects exhibited significantly increased reaction times, indicative of reduced vigilance compared to their results during the Awake scan session following a normal night's sleep (CANTAB reaction time test: $429 \pm 29>406 \pm 30 \mathrm{~ms}, \mathrm{p}=0.028$ ).

\section{Full band spectral entropy of MREG decreased during NREM sleep}

We focused our analysis of MREG spectral entropy in a ROI placed in the visual cortex (Figure 3), a brain area known to show changes in very low frequency pulsations during periods of decreased vigilance and sleep (Chang et 
al. 2016; Fukunaga et al. 2006; Liu et al. 2018). Thus, we evaluated whether MREG spectral entropy results in visual cortex can distinguish Awake and Sleep segments. As expected, the weighted sleep staging data (awake, N1, N2, N3) showed linear relationships with the scan segments $\left(A_{1}-S_{2}\right)(r=-0.952, p<0.01)$, closely resembling the association observed between the MREG spectral entropy ROI data and the scan segments $(r=-0.956, p<0.01)$ (Figure 3a-b, d). The accuracy whereby spectral entropy in the visual cortex ROI separated sleep from wakefulness was further investigated using receiver operating curve (ROC) analysis. By comparing Awake segments ( $\mathrm{n}=30$, fully awake) against Sleep ( $\mathrm{n}=30,88 \pm 12 \%$ NREM sleep), we were able to separate sleep from wakefulness with high accuracy (Area Under the Curve AUC=0.88, $\mathrm{p}<0.0001$, Figure 3f), with a sensitivity of $93 \%$ and specificity of $77 \%$. These results show that spectral entropy, which is a marker of vigilance, indeed reduced with increasing NREM sleep depth.

To analyze spatial differences in entropy between Awake and Sleep, we calculated full band voxel-wise maps in the Eyes open $\left(A_{1-2}\right)$ and Sleep segments $\left(S_{1-4}\right)$. We chose the $A_{1}$ segment for the analysis due to having the highest awake state (99\% awake, and highest vigilance based on spectral entropy ROI). We compared the Awake data versus the time series with the highest proportion of NREM sleep (91 $\pm 12 \%$ NREM sleep, $A_{1}$ versus $S_{n}$, $\left.d f 11\right)$. The occipital, parietal and temporal lobes and cerebellum exhibited significant reductions in spectral entropy (p < 0.05, df 11, Figure 3c) during NREM sleep, whereas parts of the frontal lobe did not show spectral entropy changes.

\section{Insert Figure 3 about here.}

\section{Global MREG signal revealed enhanced whole-brain pulsatility}

Next, we investigated whether the reduction in spectral entropy during NREM sleep might be linked to an increase in brain pulsation strength. FFT power of the whole-brain MREG signal was calculated to assess the strength of each pulsation. As global fMRI is known to reflect arousal fluctuations (Chang et al. 2016; Liu et al. 2018; Wen and Liu 2016), we first calculated the summed power for the global MREG spectrum. As hypothesized, the spectral power increased markedly in the very low and respiratory frequencies, and to a lesser extent in the cardiac pulsation range (Figure 4). Taken together, the power in each of the three physiological frequency bands revealed significant increases during NREM sleep compared to waking, suggesting that physiological brain pulsations are globally enhanced during sleep. 


\section{Insert Figure 4 about here.}

\section{Sleep induced entropy and power changes occurred mainly in very low and respiratory pulsations}

To investigate the spatial distribution of brain pulsations, we compared spectral power and spectral entropy maps between Awake and Sleep states, which showed significant differences mainly in very low and respiratory frequency ranges. As the power increased in very low, respiratory, and cardiac frequency bands in the transition to sleep, spectral entropy decreased in very low and respiratory bands. Figure 5 shows spectral entropy and power changes across all brain voxels, for evaluating the relative contributions of the three physiological brain pulsations during Awake and Sleep conditions.

The largest state-dependent change in power and spectral entropy occurred in the very low frequency band extending over posterior parts of the brain. The correlation coefficient between power and spectral entropy differences in Awake versus Sleep $(r=0.45)$ indicated widespread overlapping regions, especially in posterior parts of the brain. There was a power increase with no decrease in entropy in the brain stem and ventricles, and unchanged power along with low entropy values in frontal cortex. Thus, entropy can change without concomitant power changes.

The respiratory pulsation power increased and its spectral entropy decreased significantly during sleep (p $<0.05$, df 11, Figures 4-5). The power increase was located in upper posterior brain areas and in the ventricles, overlapping with the regions showing a very low frequency increase ( $r=0.34$, Figures $4-5)$. The most significant power increase occurred in typical Awake activation areas (Downar et al. 2000), thus involving the primary sensorimotor and auditory cortices, along with the V1-V2 visual areas. Power also increased in the CSF filled spaces of the lateral and central sulci, indicating strong respiratory pulsatility in sleep. In contrast to the increased respiratory frequency power, the decreased respiratory frequency spectral entropy was located more towards the basal brain structures and in the frontal pole, with less overlap with the power changes seen in upper posterior regions although still showing some overlap in visual and auditory cortices $(r=0.2)$. Spectral entropy changes were found sub-cortically near the gray matter/white matter border and more prominently in white matter, which has greater venous than arterial circulation. There was decreased spectral entropy in the brain stem, which did not overlap with power changes. Overall, very low and respiratory frequency power bands showed widespread increase during NREM sleep, in conjunction with decreased entropy in partially overlapping brain areas. 
410 411

The power of cardiac pulsation increased in somatosensory cortex during sleep ( $<<0.05$, df 11, Figure 5b, bottom), but spectral entropy did not change in the cardiac frequency range. Overall, we found that power increased in widespread brain regions for all three frequency ranges, whereas spectral entropy decreased in very low and respiratory frequency bands during NREM sleep.

\section{Insert Figure 5 about here.}

EEG slow oscillation changes overlapped with very low, respiratory and cardiac pulsation changes of

\section{MREG}

Next, we studied whether pulsation power increase and spectral entropy decrease are associated with the SWA (0.5-4 Hz) power and slow oscillation $(0.2-2 \mathrm{~Hz})$ power of EEG, as delta power changes have previously been connected with BOLD signal and CSF flow (Fultz et al. 2019) and with increased beta-amyloid clearance (Hablitz et al. 2019). The sleep induced EEG-derived slow oscillation sleep changes were located in the right middline parietal and occipital areas (Figure 6). These changes spatially overlapped with regions of increased power of all physiological brain pulsations having reduced spectral entropy. The highest overall overlap was seen in EEG slow oscillation frequency and the MREG respiratory frequency range. We did not find significant changes in SWA (0.5-4 Hz) power.

\section{Insert Figure 6 about here.}

\section{Discussion}

We studied spectral entropy and power sum changes in MREG data in very low, respiratory, and cardiac frequency bands during NREM sleep and while awake, and examined spatial similarities with concomitant EEG slow oscillation power changes. To our knowledge, this is the first study to report brain-wide enhancement and reduced spectral entropy in very low and respiratory frequency pulsations, and a lesser extent, increased cardiac frequency power. We demonstrate that regional neurophysiological changes in EEG slow oscillation power specifically colocalize with the same regions as MREG changes for all three physiological pulsations. The interaction between $0.05 \mathrm{~Hz}$ CSF pulsations with neural and hemodynamic oscillations has been shown to increase during human sleep (Fultz et al. 2019), which is supported by our present observation of these changes in mainly N2 sleep. Together 
with previous literature, our new observations suggest that the hydrodynamic properties governing CSF movement in the brain are regulated by physiological pulsations across the sleep-wake cycle.

\section{Vasomotor pulsations dominate during NREM sleep}

Present results are in accord with earlier literature on the conventional BOLD signal, which showed increased very low frequency fluctuations in posterior brain regions during light sleep and episodes of low waking vigilance (Chang et al. 2016; Fukunaga et al. 2006; Horovitz et al. 2008; Liu et al. 2018). Moreover, slow deactivation cycles of the cholinergic neurons of the nucleus basalis of Meynert have been shown to precede widely distributed hemodynamic BOLD signal increase in posterior brain regions (Liu et al. 2018). Present results show increases in very low frequency signals in posterior brain regions virtually identical to those reported by Liu et al. Spectral entropy of the EEG signal has been shown to decrease with sleep depth, where the frequency range moves towards lower frequencies and obtains a nadir during deepest sleep (Mahon et al. 2008). We found a similar pattern in MREG recordings, notably in the cerebral cortex. Therefore, we propose that the mechanism underlying the enhanced and stabilized very low frequency signal must start to dominate in the MREG 0-5 Hz frequency range.

Low frequency BOLD fluctuations are widely attributed to functionally connected hemodynamic oscillations that are tightly coupled to neuronal activity during wakefulness (Hutchison et al. 2013; Korhonen et al. 2014). Part of this low frequency activity at $\leq 0.1 \mathrm{~Hz}$ has been ascribed a vascular origin arising from vasomotor waves or slow sinusoidal hemodynamic oscillations (Biswal et al. 1995; Kiviniemi et al. 2000; Kiviniemi et al. 2016; Rayshubskiy et al. 2014; Wang et al. 2008). As, similarly shown during N2 sleep by Özbay et al. peripheral vasomotor constrictions driven by central K-complexes precede the global BOLD signal by some 11 seconds in human brain (Özbay et al. 2019). Vasomotor waves are slow, seemingly spontaneous undulations in the arteriolar wall tension that control vessel wall pulsatility and local flow resistance, which consequently influence perfusion in downstream vascular territories (Preiss and Polosa 1974). The force of the driving pulsation depends on various factors, including the circulatory perfusion pressure and elasticity of the vessel wall, both of which are regulated by smooth muscle vasomotor tonus in the arterial wall (Hadaczek et al. 2006). Recent data have indicated that vasomotor waves can move injected tracers along the perivascular space, thus serving as a glymphatic driver (van Veluw et al. 2020). 
Hillmann and colleagues have detected two distinct very low frequency phenomena using wide-field optical imaging in mice during mice (Ma et al. 2016). Urethane-anesthesia produces similar delta power activity as ketamine/xylazine anesthesia (Chauvette et al. 2011), which has been linked to increased glymphatic influx (Hablitz et al. 2019) of a similar magnitude as that occurring during natural sleep (Xie et al. 2013). A $0.04 \mathrm{~Hz}$ fluctuation present only in anesthesia data overshadowed the faster hemodynamic changes coupled to spontaneous neuronal activity (Ma et al. 2016), and only upon removing the $0.04 \mathrm{~Hz}$ vasomotor fluctuation, did the underlying neuronal activity-coupled and functionally connected hemodynamic signal emerge. Interestingly, we also found a peak power value at $0.04 \mathrm{~Hz}$ in the global MREG (Figure 4), which might arise from a similar source. Emergence of this new peaking frequency range during sleep likely decreased the spectral entropy. Plausibly, the very low frequency BOLD signal might originate from non-neuronal sources such as vasomotor activity, especially during sleep.

\section{Respiratory power increases and spectral entropy decreases during NREM sleep}

For the first time, we present evidence that sleep specific brain pulsations change in rhythm with the respiratory frequency, with increased power and reduced complexity. Although physiological pulsations can affect the BOLD signal, this interaction has not been widely studied due to technical temporal limitations of conventional fMRI (Huotari et al. 2019; Kiviniemi et al. 2016). The present acquisition of fast MREG data enable a robust separation of the cardiac and respiratory pulsations due to absence of signal aliasing over the very low frequencies (Huotari et al. 2019; Kiviniemi et al. 2016; Raitamaa et al. 2018).

During inspiration, the reduced intrathoracic pressure induces outflow of venous blood from brain that is counterbalanced by an inward movement of CSF, which is a necessary consequence of the confinement of brain within the incompressible dural venous sinuses and intracranial space (Dreha-Kulaczewski et al. 2015; Klose et al. 2000; Vinje et al. 2019; Yamada et al. 2013). This mechanism is bound also to change the perivenous CSF space, which, together with counter-phase venous blood volume changes, creates a perivenous CSF pump. In addition, pulmonary ventilation and upper airway resistance increase during sleep (Sowho et al. 2014; Trinder et al. 1992; Wiegand et al. 1989; Worsnop et al. 1998). The increased intrathoracic ventilation pressures may consequently promote the (peri)venous CSF pumping action in the brain, manifesting in increased respiratory frequency power in the MREG signal (Dreha-Kulaczewski et al. 2015; Matsumae et al. 2019; Vinje et al. 2019). In addition, our results showing decreased spectral entropy in the respiratory frequency range during N1-2 sleep are in accord with 
findings that the respiratory pattern is more regular during NREM sleep compared with waking (Malik et al. 2012). The increased and stabilized physiological pulsations during sleep would likely increase CSF flow towards the neuropil, which might inflate the extracellular space, as has been shown to occur during sleep/wake transitions (Xie et al. 2013).

Furthermore, the increased respiratory and very low frequency brain pulsations in NREM sleep overlap in posterior brain regions, which may be mediated by shared autonomic pressure control mechanisms (Özbay et al. 2019). In line with previous literature (Ma et al. 2016; Özbay et al. 2019), we suppose that the physiological pulsations may overpower neurovascularly coupled activity in the primary sensory processing areas during mainly N2 sleep. Thus, the enhanced brain clearance in light N2 sleep may arise in cortical areas that process sensory information in the awake state (Downar et al. 2000).

\section{Cardiovascular pulsations enhance in somatosensory cortex}

Microscopic studies in rodent brain have shown that parenchymal cardiovascular pulsatility is the main contributor to CSF convection (Iliff et al. 2013; Mestre et al. 2018), whereas our whole brain analysis results do not show large scale changes during NREM sleep. Cardiovascular pulsatility provides energy substrates supporting neuronal activity, which overall stays stable. However, we saw increased cardiac power in somatosensory cortex, a region that is known to remain active during sleep (Ramot et al. 2013). Lecci et al. found that a $0.02 \mathrm{~Hz}$ oscillation occurred in somatosensory cortex in NREM sleep in mice and humans, and went on to suggest that such oscillations are mainly caused by sleep spindles tightly coupled with heart rate (Lecci et al. 2017). While the present data suggest that vasomotor and respiratory pulsations dominate throughout the neuropil during sleep, the interactions between the physiological pulsations need to be further investigated.

\section{Coupling between EEG and MREG pulsations}

Recent studies have shown that the delta power changes in EEG are tightly related to BOLD activity changes along with faster CSF flow (Fultz et al. 2019), and that higher influx of CSF in the brain parenchyma allows increased solute transport from the brain (Hablitz et al. 2019). Fultz et al. showed that the onset of slow-delta EEG occurred 6.4 seconds prior to the CSF peak, and concluded that such coupling was strongest during sleep (Fultz et al. 2019). In addition, they found that BOLD and CSF signals were anticorrelated in the cortical grey matter, thus suggesting an alternation between BOLD and CSF flow, in accord with the constraint of constant intracranial volume. 
Interestingly, recent work showed that controlled deep inspirations during wakefulness were followed by fMRI and CSF changes similar to those occurring during NREM 1 and 2 sleep, thus suggesting that respiration is actually a driver for EEG and fMRI signal (Picchioni et al. 2021). Along the same lines, an increased respiratory effect on neuronal brain activity has been detected in intracranial needle measurements and MREG studies of epileptic patients (Herrero et al. 2018; Kananen et al. 2020; Kananen et al. 2018; Zelano et al. 2016). Our findings suggest that the main changes in EEG occurred in slow oscillation power, which increased in concert with higher pulsation strength and reduced spectral entropy. Mechanistically, slow oscillation power increases along with elevated interstitial electrolyte levels and narrowing of the intercellular space (Ding et al. 2016; Hablitz et al. 2019; Xie et al. 2013); such mechanisms are likely to also occur during increased SWA human sleep. Since pulsation changes appeared in early sleep (NREM 1-2) and overlapped with EEG slow oscillation changes, we argue that these findings could reflect a direct physiological interaction between electrohydrodynamic pulsations rather than arising from an interaction with the faster neurophysiological pulsations that markedly decline in power during sleep.

\section{Limitations}

The Awake scan was performed at 4-6 PM and the Sleep scan at 6-8 AM, such that circadian rhythms might have added a confound to our results. To minimize that possibility, we selected for analysis those segments that included the highest amount of sleep according to AASM sleep scoring criteria. In terms of naturalistic sleep, the fMRI laboratory is a challenging environment for attaining deep sleep; unfortunately, we only observed one epoch of N3 sleep. We conducted the sleep deprivation prior to the Sleep scan session to help subjects fall asleep in the scanner. While somewhat effective, this intervention may have increased sympathetic activity as is described to occur after sleep deprivation (Tobaldini et al. 2017). The sleep deprivation took place at home instead of the laboratory environment, such that we are reliant upon surrogate measures of sleep deprivation in some subjects.

We did not perform screening for sleep apnea or other sleep disorders since all included subjects were young and generally healthy. Our sample size was small $(n=12-15)$ calling for replication of some analysis in additional subjects. Subjective sleep quality was not assessed with validated questionnaires which we consider to be another limitation. There was no a priori statistical power analysis. We had no criteria for the eating schedule for the subjects. Further work is required to investigate the exact source of different physiological changes over the whole 0.008-5 Hz frequency band that is accessible with direct current EEG and MREG. 
544

\section{Conclusions}

NREM sleep alters all three physiological brain pulsations, with the greatest power increases and spectral entropy decreases in the vasomotor and respiratory pulsations. In addition, we found small power increase in cardiac frequency band. Electrophysiological slow oscillation $\quad$ power increased in brain regions overlapping with all pulsation changes, suggesting that these pulsations participate in sleep-related electrophysiological brain processes with an inverse relation to pulsation frequency. This inverse frequency change robustly reduces entropy of the brain physiological signal spectrum, enabling a clear dissociation of sleep and awake states from MREG data. We suggest the physiological brain pulsations alongside slow wave electrophysiological changes in NREM sleep likely contribute to elevated CSF flow.

\section{References}

Allen PJ, Josephs O, Turner R (2000) A method for removing imaging artifact from continuous EEG recorded during functional MRI. Neuroimage 12:230-239.

Allen PJ, Polizzi G, Krakow K, Fish DR, Lemieux L (1998) Identification of EEG events in the MR scanner: The problem of pulse artifact and a method for its subtraction. Neuroimage 8:229-239.

Assländer J, Zahneisen B, Hugger T, Reisert M, Lee H-, LeVan P, Hennig J (2013) Single shot whole brain imaging using spherical stack of spirals trajectories. Neuroimage 73:59-70.

Berger H (1901) Zur Lehre von der Blutzirkulation in der Schädelhöhle des Menschen namentlich unter dem Einfluss von Medikamenten. Jena: Verlag von Gustav Fischer, .

Biswal B, Zerrin Yetkin F, Haughton VM, Hyde JS (1995) Functional connectivity in the motor cortex of resting human brain using echo-planar mri. Magn Reson Med 34:537-541.

Brancaccio A, Tabarelli D, Bigica M, Baldauf D (2020) Cortical source localization of sleep-stage specific oscillatory activity. Sci Rep 10:6976.

CANTAB ${ }^{\circledR}$ (2019) [Cognitive assessment software]. Cambridge Cognition. All rights reserved. www.cantab.com. 
Chang C, Leopold DA, Schölvinck ML, Mandelkow H, Picchioni D, Liu X, Ye FQ, Turchi JN, Duyn JH (2016) Tracking brain arousal fluctuations with fMRI. Proc Natl Acad Sci U S A 113:4518-4523.

Chauvette S, Crochet S, Volgushev M, Timofeev I (2011) Properties of slow oscillation during slow-wave sleep and anesthesia in cats. J Neurosci 31:14998-15008.

Cox RW (1996) AFNI: Software for analysis and visualization of functional magnetic resonance neuroimages. Comput Biomed Res 29:162-173.

de Zambotti M, Rosas L, Colrain IM, Baker FC (2017) The Sleep of the Ring: Comparison of the OURA Sleep Tracker Against Polysomnography. Behav Sleep Med 17:1-15.

Ding F, O'donnell J, Xu Q, Kang N, Goldman N, Nedergaard M (2016) Changes in the composition of brain interstitial ions control the sleep-wake cycle. Sci 352:550-555.

Downar J, Crawley AP, Mikulis DJ, Davis KD (2000) A multimodal cortical network for the detection of changes in the sensory environment. Nat Neurosci 3:277-283.

Dreha-Kulaczewski S, Joseph AA, Merboldt K-, Ludwig H-, Gärtner J, Frahm J (2015) Inspiration is the major regulator of human CSF flow. J Neurosci 35:2485-2491.

Duff EP, Johnston LA, Xiong J, Fox PT, Mareels I, Egan GF (2008) The power of spectral density analysis for mapping endogenous BOLD signal fluctuations. Hum Brain Mapp 29:778-790.

Fukunaga M, Horovitz SG, van Gelderen P, de Zwart JA, Jansma JM, Ikonomidou VN, Chu R, Deckers RHR, Leopold DA, Duyn JH (2006) Large-amplitude, spatially correlated fluctuations in BOLD fMRI signals during extended rest and early sleep stages. Magn Reson Imaging 24:979-992.

Fultz NE, Bonmassar G, Setsompop K, Stickgold RA, Rosen BR, Polimeni JR, Lewis LD (2019) Coupled electrophysiological, hemodynamic, and cerebrospinal fluid oscillations in human sleep. Sci 366:628-631.

Hablitz LM, Vinitsky HS, Sun Q, Staeger FF, Sigurdsson B, Mortensen KN, Lilius TO, Nedergaard M (2019) Increased glymphatic influx is correlated with high EEG delta power and low heart rate in mice under anesthesia. Sci Adv 5:2.

Hadaczek P, Yamashita Y, Mirek H, Tamas L, Bohn MC, Noble C, Park JW, Bankiewicz K (2006) The "Perivascular Pump" Driven by Arterial Pulsation Is a Powerful Mechanism for the Distribution of Therapeutic Molecules within the Brain. Mol Ther 14:69-78.

Herrero JL, Khuvis S, Yeagle E, Cerf M, Mehta AD (2018) Breathing above the brain stem: Volitional control and attentional modulation in humans. J Neurophysiol 119:145-159.

Horovitz SG, Braun AR, Carr WS, Picchioni D, Balkin TJ, Fukunaga M, Duyn JH (2009) Decoupling of the brain's default mode network during deep sleep. Proc Natl Acad Sci U S A 106:11376-11381.

Horovitz SG, Fukunaga M, De Zwart JA, Van Gelderen P, Fulton SC, Balkin TJ, Duyn JH (2008) Low frequency BOLD fluctuations during resting wakefulness and light sleep: A simultaneous EEG-fMRI study. Hum Brain Mapp 29:671-682.

Hugger T, Zahneisen B, LeVan P, Lee KJ, Lee H-, Zaitsev M, Hennig J (2011) Fast undersampled functional magnetic resonance imaging using nonlinear regularized parallel image reconstruction. PLoS ONE 6:12.

Huotari N, Raitamaa L, Helakari H, Kananen J, Raatikainen V, Rasila A, Tuovinen T, Kantola J, Borchardt V, Kiviniemi VJ, Korhonen VO (2019) Sampling rate effects on resting state fMRI metrics. Front Neurosci 13:279. 
612 Hutchison RM, Womelsdorf T, Allen EA, Bandettini PA, Calhoun VD, Corbetta M, Della Penna S, Duyn JH,

613 Glover GH, Gonzalez-Castillo J, Handwerker DA, Keilholz S, Kiviniemi V, Leopold DA, de Pasquale F, Sporns O, 614 Walter M, Chang C (2013) Dynamic functional connectivity: Promise, issues, and interpretations. Neuroimage 615 80:360-378.

616 Iliff JJ, Wang M, Zeppenfeld DM, Venkataraman A, Plog BA, Liao Y, Deane R, Nedergaard M (2013) Cerebral 617 arterial pulsation drives paravascular CSF-Interstitial fluid exchange in the murine brain. J Neurosci 33:1819061818199.

Jenkinson M, Beckmann CF, Behrens TEJ, Woolrich MW, Smith SM (2012) FSL. Neuroimage 62:782-790.

Jenkinson M, Bannister P, Brady M, Smith S (2002) Improved optimization for the robust and accurate linear registration and motion correction of brain images. Neuroimage 17:825-841.

Kananen J, Helakari H, Korhonen V, Huotari N, Järvelä M, Raitamaa L, Raatikainen V, Rajna Z, Tuovinen T, Nedergaard M, Jacobs J, LeVan P, Ansakorpi H, Kiviniemi V (2020) Respiratory-related brain pulsations are

Kananen J, Tuovinen T, Ansakorpi H, Rytky S, Helakari H, Huotari N, Raitamaa L, Raatikainen V, Rasila A, Borchardt V, Korhonen V, LeVan P, Nedergaard M, Kiviniemi V (2018) Altered physiological brain variation in drug-resistant epilepsy. Brain Behav 8:9.

Kaufmann C, Wehrle R, Wetter TC, Holsboer F, Auer DP, Pollmächer T, Czisch M (2006) Brain activation and hypothalamic functional connectivity during human non-rapid eye movement sleep: An EEG/fMRI study. Brain 129:655-667.

Kiviniemi V, Wang X, Korhonen V, Keinänen T, Tuovinen T, Autio J, Levan P, Keilholz S, Zang Y-, Hennig J, Nedergaard M (2016) Ultra-fast magnetic resonance encephalography of physiological brain activity-Glymphatic pulsation mechanisms?. J Cereb Blood Flow Metab 36:1033-1045.

Kiviniemi V, Jauhiainen J, Tervonen O, Pääkkö E, Oikarinen J, Vainionpää V, Rantala H, Biswal B (2000) Slow vasomotor fluctuation in fMRI of anesthetized child brain. Magn Reson Med 44:373-378.

Klose U, Strik C, Kiefer C, Grodd W (2000) Detection of a relation between respiration and CSF pulsation with an echoplanar technique. J Magn Reson Imaging 11:438-444.

Korhonen V, Hiltunen T, Myllylä T, Wang X, Kantola J, Nikkinen J, Zang Y-, LeVan P, Kiviniemi V (2014) Synchronous multiscale neuroimaging environment for critically sampled physiological analysis of brain function: hepta-scan concept. Brain Connect 4:677-689.

Kumar R, Ramaswamy R, Nath Mallick B (2013) Local Properties of Vigilance States: EMD Analysis of EEG Signals during Sleep-Waking States of Freely Moving Rats. PLoS ONE 8:10.

Lecci S, Fernandez LMJ, Weber FD, Cardis R, Chatton J-, Born J, Lüthi A (2017) Coordinated infraslow neural and cardiac oscillations mark fragility and offline periods in mammalian sleep. Sci Adv 3:2.

Lee H-, Zahneisen B, Hugger T, LeVan P, Hennig J (2013) Tracking dynamic resting-state networks at higher frequencies using MR-encephalography. Neuroimage 65:216-222.

Liu X, De Zwart JA, Schölvinck ML, Chang C, Ye FQ, Leopold DA, Duyn JH (2018) Subcortical evidence for a contribution of arousal to fMRI studies of brain activity. Nat Commun 9, 395.

Ma Y, Shaik MA, Kozberg MG, Kim SH, Portes JP, Timerman D, Hillman EMC (2016) Resting-state hemodynamics are spatiotemporally coupled to synchronized and symmetric neural activity in excitatory neurons. Proc Natl Acad Sci U S A 113:E8463-E8471. 
Mahon P, Greene BR, Lynch EM, McNamara B, Shorten GD (2008) Can state or response entropy be used as a measure of sleep depth? Anaesthesia 63:1309-1313.

Malik V, Smith D, Lee-Chiong Jr. T (2012) Respiratory physiology during sleep . Sleep Med Clin 7:497-505.

Mason JW, Ramseth DJ, Chanter DO, Moon TE, Goodman DB, Mendzelevski B (2007) Electrocardiographic reference ranges derived from 79,743 ambulatory subjects. J Electrocardiol 40:228-234.e8.

Matsumae M, Kuroda K, Yatsushiro S, Hirayama A, Hayashi N, Takizawa K, Atsumi H, Sorimachi T (2019) Changing the currently held concept of cerebrospinal fluid dynamics based on shared findings of cerebrospinal fluid motion in the cranial cavity using various types of magnetic resonance imaging techniques. Neurol Med -Chir 59:133-146.

Mehrabadi MA, Azimi I, Sarhaddi F, Axelin A, Niela-Vilén H, Myllyntausta S, Stenholm S, Dutt N, Liljeberg P, Rahmani AM (2020) Sleep tracking of a commercially available smart ring and smartwatch against medical-grade actigraphy in everyday settings: Instrument validation study. JMIR mHealth uHealth 8:10.

Mestre H, Tithof J, Du T, Song W, Peng W, Sweeney AM, Olveda G, Thomas JH, Nedergaard M, Kelley DH (2018) Flow of cerebrospinal fluid is driven by arterial pulsations and is reduced in hypertension. Nat Commun 9:4878.

Özbay PS, Chang C, Picchioni D, Mandelkow H, Chappel-Farley MG, van Gelderen P, de Zwart JA, Duyn J (2019) Sympathetic activity contributes to the fMRI signal. Commun Biolog 2:1.

Penzel T, Kantelhardt JW, Lo C-, Voigt K, Vogelmeier C (2003) Dynamics of heart rate and sleep stages in normals and patients with sleep apnea. Neuropsychopharmacology 28:S48-S53.

Picchioni D, Özbay PS, Mandelkow H, Zwart JA, Wang Y, Gelderen P, Duyn J (2021) Autonomic arousals contribute to brain fluid pulsations during sleep. BioRxiv doi: https://doi.org/10.1101/2021.05.04.442672.

Posse S, Ackley E, Mutihac R, Zhang T, Hummatov R, Akhtari M, Chohan M, Fisch B, Yonas H (2013) Highspeed real-time resting-state fMRI using multi-slab echo-volumar imaging. Front Human Neurosci 7:479.

Preiss G, Polosa C (1974) Patterns of sympathetic neuron activity associated with Mayer waves. Am J Physiol 226:724-730.

Raitamaa L, Korhonen V, Huotari N, Raatikainen V, Hautaniemi T, Kananen J, Rasila A, Helakari H, Zienkiewicz A, Myllylä T, Borchardt V, Kiviniemi V (2018) Breath hold effect on cardiovascular brain pulsations - A multimodal magnetic resonance encephalography study. J Cereb Blood Flow Metab 39:2471-2485.

Ramot M, Fisch L, Davidesco I, Harel M, Kipervasser S, Andelman F, Neufeld MY, Kramer U, Fried I, Malach R (2013) Emergence of sensory patterns during sleep highlights differential dynamics of REM and non-REM sleep stages. J Neurosci 33:14715-14728.

Rayshubskiy A, Wojtasiewicz TJ, Mikell CB, Bouchard MB, Timerman D, Youngerman BE, McGovern RA, Otten ML, Canoll P, McKhann GM, Hillman EMC (2014) Direct, intraoperative observation of $\sim 0.1 \mathrm{~Hz}$ hemodynamic oscillations in awake human cortex: Implications for fMRI. Neuroimage 87:323-331.

Russo MA, Santarelli DM, O'Rourke D (2017) The physiological effects of slow breathing in the healthy human. Breathe 13:298-309.

Shannon CE (1948) The Mathematical Theory of Communication. Bell System Technical Journal 27:379-423, 623-656.

Silvani A, Bojic T, Franzini C, Lenzi P, Walker AM, Grant DA, Wild J, Zoccoli G (2004) Sleep-related changes in the regulation of cerebral blood flow in newborn lambs. Sleep 27:36-41. 
Sloots JJ, Biessels GJ, Zwanenburg JJM (2020) Cardiac and respiration-induced brain deformations in humans quantified with high-field MRI. Neuroimage 210:116581.

Smith SM (2002) Fast robust automated brain extraction. Hum Brain Mapp 17:143-155.

Sowho M, Amatoury J, Kirkness JP, Patil SP (2014) Sleep and respiratory physiology in adults. Clin Chest Med 35:469-481.

Tait MJ, Saadoun S, Bell BA, Papadopoulos MC (2008) Water movements in the brain: role of aquaporins. Trends Neurosci 31:37-43.

Tobaldini E, Costantino G, Solbiati M, Cogliati C, Kara T, Nobili L, Montano N (2017) Sleep, sleep deprivation, autonomic nervous system and cardiovascular diseases. Neurosci Biobehav Rev 74:321-329.

Trinder J, Whitworth F, Kay A, Wilkin P (1992) Respiratory instability during sleep onset. J Appl Physiol 73:24622469.

Tuovinen T, Kananen J, Rajna Z, Lieslehto J, Korhonen V, Rytty R, Mattila H, Huotari N, Raitamaa L, Helakari H, Elseoud AA, Krüger J, LeVan P, Tervonen O, Hennig J, Remes AM, Nedergaard M, Kiviniemi V (2020) The variability of functional MRI brain signal increases in Alzheimer's disease at cardiorespiratory frequencies. Sci Rep 10:1.

Vakkuri A, Yli-Hankala A, Sandin R, Mustola S, Høymork S, Nyblom S, Talja P, Sampson T, Van Gils M, Viertiö-Oja H (2005) Spectral entropy monitoring is associated with reduced propofol use and faster emergence in propofol-nitrous oxide-alfentanil anesthesia. Anesthesiology 103:274-279.

van Veluw SJ, Hou SS, Calvo-Rodriguez M, Arbel-Ornath M, Snyder AC, Frosch MP, Greenberg SM, Bacskai BJ (2020) Vasomotion as a Driving Force for Paravascular Clearance in the Awake Mouse Brain. Neuron 105:549561.

Viertiö-Oja H, Maja V, Särkelä M, Talja P, Tenkanen N, Tolvanen-Laakso H, Paloheimo M, Vakkuri A, YliHankala A, Meriläinen P (2004) Description of the Entropy ${ }^{\mathrm{TM}}$ algorithm as applied in the Datex-Ohmeda $5 / 5^{\mathrm{TM}}$ Entropy Module. Acta Anaesthesiol Scand 48:154-161.

Vinje V, Ringstad G, Lindstrøm EK, Valnes LM, Rognes ME, Eide PK, Mardal K- (2019) Respiratory influence on cerebrospinal fluid flow - a computational study based on long-term intracranial pressure measurements. Sci Rep 9:1.

Wang HH, Menezes NM, Ming WZ, Ay H, Koroshetz WJ, Aronen HJ, Karonen JO, Liu Y, Nuutinen J, Wald LL, Sorensen AG (2008) Physiological noise in MR images: An indicator of the tissue response to ischemia?. J Magn Reson Imaging 27:866-871.

Wen H, Liu Z (2016) Broadband electrophysiological dynamics contribute to global resting-state fMRI signal. J Neurosci 36:6030-6040.

Wiegand L, Zwillich CW, White DP (1989) Collapsibility of the human upper airway during normal sleep. J Appl Physiol 66:1800-1808.

Wong CW, DeYoung PN, Liu TT (2016) Differences in the resting-state fMRI global signal amplitude between the eyes open and eyes closed states are related to changes in EEG vigilance. Neuroimage 124:24-31.

Worsnop C, Kay A, Pierce R, Kim Y, Trinder J (1998) Activity of respiratory pump and upper airway muscles during sleep onset. J Appl Physiol 85:908-920.

Xie L, Kang H, Xu Q, Chen MJ, Liao Y, Thiyagarajan M, O'Donnell J, Christensen DJ, Nicholson C, Iliff JJ, Takano T, Deane R, Nedergaard M (2013) Sleep drives metabolite clearance from the adult brain. Sci 342:373-377. 
Yamada S, Miyazaki M, Yamashita Y, Ouyang C, Yui M, Nakahashi M, Shimizu S, Aoki I, Morohoshi Y, McComb JG (2013) Influence of respiration on cerebrospinal fluid movement using magnetic resonance spin labeling. Fluids Barriers CNS 10:1.

Zahneisen B, Hugger T, Lee KJ, Levan P, Reisert M, Lee H-, Assländer J, Zaitsev M, Hennig J (2012) Single shot concentric shells trajectories for ultra fast fMRI. Magn Reson Med 68:484-494.

Zelano C, Jiang H, Zhou G, Arora N, Schuele S, Rosenow J, Gottfried JA (2016) Nasal respiration entrains human limbic oscillations and modulates cognitive function. J Neurosci 36:12448-12467.

\section{Figure legends}

Figure 1. Data processing and exclusion of subjects. 25 subjects were recruited and scanned in the study. The final analyses included 15 and 12 subjects, whose results are presented in the manuscript.

Figure 2. Study protocol and exemplary signals of awake state and NREM sleep. a. Study design through one week of sleep monitoring. Awake scanning session was performed on day two after a good night's sleep. Sleep scanning session was performed on day five, the morning after a night of documented sleep deprivation. At the beginning of both sessions, subjects underwent a CANTAB test to evaluate reaction times. In the Awake scan session, two scans were recorded: I) eyes open $\left(\mathrm{A}_{1-2}\right)$ and II) eyes closed (EC). In the Sleep scan session two scans were taken: I) ten-minute Sleep scan $\left(\mathrm{S}_{1-2}\right)$ and $\left.\mathrm{II}\right)$ ten-minute Sleep scan $\left(\mathrm{S}_{3-4}\right)$. Numbers $\left(\mathrm{A}_{1-2}, \mathrm{~S}_{1-4}\right)$ corresponding to five-minute segments that were treated separately for further analysis. Percent represents the average fraction of 
sleep during the scan segment. b-e. Examples of electrophysiological and dynamic brain pulsations measured in the same subject while awake (100\% awake) and sleeping (80\% NREM stage 2, 20\% NREM stage 1) for five-minute recordings. Thus, the power spectra were calculated for an entire five-minute segment. The data were measured simultaneously by b-c. 256 lead high density DC-EEG (Oz) and d-e. fast $10 \mathrm{~Hz}$ MREG covering the whole brain in the $\leq 5 \mathrm{~Hz}$ band. Both time and frequency domain data indicated increased power and amplitude of brain pulsations upon transition from the EEG-verified fully awake state to NREM sleep. The dominant increase in very low frequency pulsation power reshapes the power spectrum distribution and alters the spectral entropy of the EEG and MREG signals.

Figure 3. Spectral entropy of MREG decreases in line with increased amount of NREM sleep. a. EEG sleep state data show that the amount and depth of sleep both increase as a function of scan segment. b. Visual cortex $\mathrm{SE}_{\mathrm{ROI}}$ of MREG data predicts sleep and wakefulness across subjects $(\mathrm{n}=12)$. Results indicate linear declines both in EEG sleep state and MREG $\mathrm{SE}_{\mathrm{ROI}}$ values as a function of scan segment in the experiment. c. Spectral entropy decreased significantly in posterior brain areas $(\mathrm{p}<0.05$, df 11$)$. $\mathrm{d}$. The $\mathrm{SE}_{\mathrm{ROI}}$ showed a drop in all subjects in the transition from wakefulness to EEG-verified sleep states in $S_{2}$. $f$. The ROC curve of $\mathrm{SE}_{\mathrm{ROI}}$ data indicate high accuracy AUC $=0.88(\mathrm{p}<0.0001)$ in the ability to distinguish sleep $(\mathrm{n}=30$ Sleep segments $)$ from awake data $(\mathrm{n}=$ 30 Awake segments). The model has a sensitivity of $93 \%$ and a specificity of $77 \%$.

Figure 4. NREM sleep sharply changes the power spectrum of physiological brain pulsations. Global MREG power spectra and power sum difference (paired t-test) between Awake and Sleep in: a. very low frequency (0.008$0.1 \mathrm{~Hz})$, b. respiratory frequency $(0.11-0.44 \mathrm{~Hz})$, and c. cardiac frequency $(0.52-1.6 \mathrm{~Hz})$ bands. The global very low and respiratory frequency power both increased markedly in sleep, and both differed significantly in posterior brain regions. The global cardiac frequency power increased to a lesser extent, but showed decrease in heart rate $(\mathrm{p}<$ $\left.0.01^{* *}, \mathrm{p}<0,05^{*}, \mathrm{df} 11\right)$.

Figure 5. Spatial distribution of spectral brain pulsation entropy and power are linked to frequency band. Paired t-test shows differences in Sleep versus Awake state $(\mathrm{p}<0.05$, df 11$)$. Thresholded mean maps across subjects and difference maps are presented. A. spectral entropy and b. spectral power in each pulsations frequency band in five-minute segments in NREM sleep. The three rows show results in very low, respiratory and cardiac frequencies, respectively. The spatial overlap of the power and entropy increases dimishes as a function of 
frequency, with largest overlap in the very low frequency range, partial overlap in the respiratory band, and no overlap in cardiac frequencies (no change in spectral entropy).

Figure 6. EEG slow oscillation $(0.2-2 \mathrm{~Hz})$ power increased in spatially overlapping regions in very low, respiratory and cardiac frequency pulsations in MREG. a. EEG slow oscillation (0.2-2 Hz) power sum mean maps in Awake and Sleep, and difference (Sleep > Awake, $\mathrm{p}<0.05$ ). EEG power maps were converted to the radiological perspective for comparison with MREG results. b. Power sum of MREG pulsations in very low frequency $(0.008-0.1 \mathrm{~Hz})$, c. respiratory frequency $(0.11-0.44 \mathrm{~Hz})$ and d. cardiac frequency $(0.52-1.6 \mathrm{~Hz})$, Sleep > Awake, $\mathrm{p}<0.05$. e. Spectral entropy of MREG in very low frequency $(0.008-0.1 \mathrm{~Hz})$, Sleep $<$ Awake, $<<0.05$. Mean maps between subjects during Awake and Sleep are placed on the left and difference maps on the right column.

\section{Tables}

Table 1. The amount of NREM sleep was higher during the Sleep scan session than in the Awake scan session. Mean \pm standard deviation $(\%)$ of the amounts of wakefulness, NREM and total sleep times were calculated from the number of sleep-scored EEG epochs in each condition. The highest amount of sleep was achieved in $\mathrm{S}_{2}$ (mean across subjects 87\%). Unknown epochs are likely due to artefacts. $\mathrm{A}_{1}$ (Awake 1 time segment, five min); $\mathrm{A}_{2}$ (Awake 2 time segment, five min); EC (Eyes closed, five min); $\mathrm{S}_{1}$ (Sleep 1 time segment, five $\mathrm{min}$ ); $\mathrm{S}_{2}$ (Sleep 2 time segment, five $\mathrm{min}$ ); $\mathrm{S}_{3}$ (Sleep 3 time segment, five $\mathrm{min}$ ); $\mathrm{S}_{4}$ (Sleep 4 time segment, five min). $\mathrm{N}$ values represent the number of totally 15 subjects who had available EEG for sleep scoring.

\begin{tabular}{|c|c|c|c|c|c|c|c|}
\hline \multicolumn{4}{|c|}{ Awake scan session } & \multicolumn{4}{|c|}{ Sleep scan session } \\
\hline Mean \pm STD & $\mathrm{A}_{1}$ & $\mathrm{~A}_{2}$ & EC & $\overline{\mathrm{S}_{1}}$ & $\mathrm{~S}_{2}$ & $\mathrm{~S}_{3}$ & $\mathrm{~S}_{4}$ \\
\hline$(\%)$ & $\mathrm{n}=12$ & $\mathrm{n}=12$ & $\mathrm{n}=13$ & $\mathrm{n}=12$ & $\mathrm{n}=12$ & $\mathrm{n}=10$ & $\mathrm{n}=10$ \\
\hline Wake & $99 \pm 3$ & $99 \pm 3$ & $68 \pm 39$ & $22 \pm 19$ & $11 \pm 23$ & $23 \pm 32$ & $22 \pm 35$ \\
\hline N1 & $1 \pm 3$ & $1 \pm 3$ & $28 \pm 39$ & $47 \pm 25$ & $40 \pm 33$ & $40 \pm 22$ & $35 \pm 30$ \\
\hline N2 & 0 & 0 & $4 \pm 14$ & $25 \pm 19$ & $46 \pm 38$ & $37 \pm 29$ & $37 \pm 36$ \\
\hline N3 & 0 & 0 & 0 & 0 & $1 \pm 3$ & 0 & 0 \\
\hline
\end{tabular}


No REM sleep was observed.

\begin{tabular}{llllllll}
\hline NREM & sleep & & & & & & \\
(\%) & $1 \pm 3$ & $1 \pm 3$ & $32 \pm 40$ & $72 \pm 17$ & $87 \pm 21$ & $77 \pm 30$ & $72 \pm 35$
\end{tabular}

810 Table 2. Respiratory and cardiac frequency ranges were determined from physiological $\mathrm{EtCO}_{2}$ and $\mathrm{SpO}_{2}$

811 signals. Individually determined minimum (min) and maximum (max) values for lower and upper edge of the 812 power peak were used for the further analysis. Respiratory rate and heart rate decreased in NREM sleep versus 813 awake based on the power peaking value.

Respiratory $\mathrm{EtCO}_{2}(\mathrm{~Hz})$

\begin{tabular}{lllll}
\hline & & Min & Peak & Max \\
Eyes open & $\mathrm{n}=14$ & $0.17 \pm 0.06$ & $0.27 \pm 0.06$ & $0.35 \pm 0.05$ \\
Eyes closed & $\mathrm{n}=13$ & $0.13 \pm 0.06$ & $0.24 \pm 0.06$ & $0.32 \pm 0.05$ \\
Sleep scan 1 & $\mathrm{n}=14$ & $0.17 \pm 0.05$ & $0.24 \pm 0.04^{*}$ & $0.31 \pm 0.05$ \\
Sleep scan 2 & $\mathrm{n}=12$ & $0.16 \pm 0.05$ & $0.24 \pm 0.05^{*}$ & $0.32 \pm 0.06$ \\
\hline & & & \\
& & & & \\
& & & \\
& & & \\
& & & \\
Eyes open & $\mathrm{n}=14$ & $0.96 \pm 0.10$ & $1.07 \pm 0.12$ & $1.19 \pm 0.16$ \\
Eyes closed & $\mathrm{n}=13$ & $0.90 \pm 0.12$ & $1.03 \pm 0.13^{* * *}$ & $1.19 \pm 0.18$ \\
Sleep scan 1 & $\mathrm{n}=14$ & $0.81 \pm 0.13$ & $0.94 \pm 0.16^{* *}$ & $1.09 \pm 0.17$ \\
Sleep scan 2 & $\mathrm{n}=11$ & $0.82 \pm 0.12$ & $0.94 \pm 0.16$ & $1.11 \pm 0.21$
\end{tabular}


2 subjects: apneic breathing patterns

1 subject:

excessive motion and insufficient sleep prior to the Awake scan

\section{2 subjects}

EEG preprocessing EEG sleep scoring

(AASM criteria)

Exclusion of

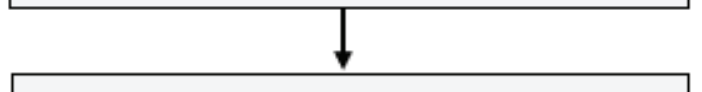

15 subjects

CANTAB and Smart ring analysis

MREG analysis:

Exclusion of

Spectral entropy ROI accuracy

\section{2 subjects}

Paired MREG analysis:

Spectral entropy

Spectral power sum

EEG slow oscillation analysis
3 subjects:

EEG artifact prohibited sleep scoring from sleep data 


\section{a. Study protocol}

\begin{tabular}{|c|c|c|c|c|c|c|c|c|c|c|c|c|c|}
\hline \multicolumn{14}{|c|}{ Sleep tracking } \\
\hline Day1 & \multicolumn{4}{|c|}{ Day2 } & Day3 & Day4 & \multicolumn{5}{|c|}{ Day5 } & Day6 & Day7 \\
\hline & \multicolumn{4}{|c|}{ 4-6 PM Awake scan session } & \multirow{3}{*}{\multicolumn{2}{|c|}{$\begin{array}{c}\text { Night4 } \\
\text { Sleep } \\
\text { deprivation }\end{array}$}} & \multicolumn{5}{|c|}{ Sleep scan session } & & \\
\hline & \multirow[b]{2}{*}{$\begin{array}{c}\text { Cantab } \\
\text { test }\end{array}$} & Scar & & Scan 2 & & & \multirow[b]{2}{*}{$\begin{array}{c}\text { Cantab } \\
\text { test }\end{array}$} & \multicolumn{2}{|c|}{ Scan 1} & \multicolumn{2}{|c|}{ Scan 2} & & \\
\hline & & \begin{tabular}{c|}
$+2)$ \\
$A_{1}$ \\
$1 \%)$
\end{tabular} & $\begin{array}{l}+2) \\
\mathrm{A}_{2} \\
1(\%)\end{array}$ & $\begin{array}{c}\lambda_{\text {NC }} \\
\text { EC } \\
(32 \%)\end{array}$ & & & & ${\stackrel{\mathrm{S}}{\mathrm{S}_{1}^{\mathrm{z}^{\mathrm{z}}}}}_{(72 \%)}$ & $\begin{array}{c}\mathrm{z}^{\mathrm{z}^{\mathrm{z}}} \\
\mathrm{S}_{2} \\
(87 \%)\end{array}$ & $\mid \begin{array}{c}\mathrm{Z}^{\mathrm{Z}^{2}} \\
\mathrm{~S}_{3} \\
(77 \%)\end{array}$ & \begin{tabular}{|c|}
$\mathrm{Z}^{\mathrm{z}^{\mathrm{z}}}$ \\
$\mathrm{S}_{4}^{\mathrm{S}}$ \\
$(72 \%)$ \\
\end{tabular} & & \\
\hline
\end{tabular}

\section{b. Awake EEG}

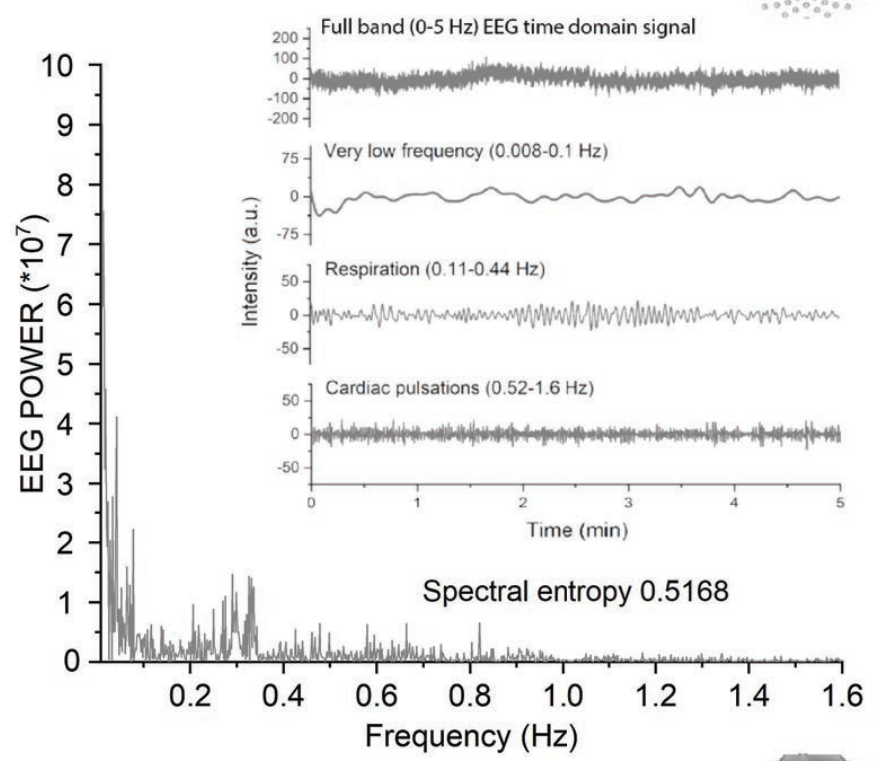

\section{d. Awake MREG}

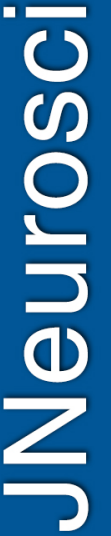

\section{c. Sleep EEG}

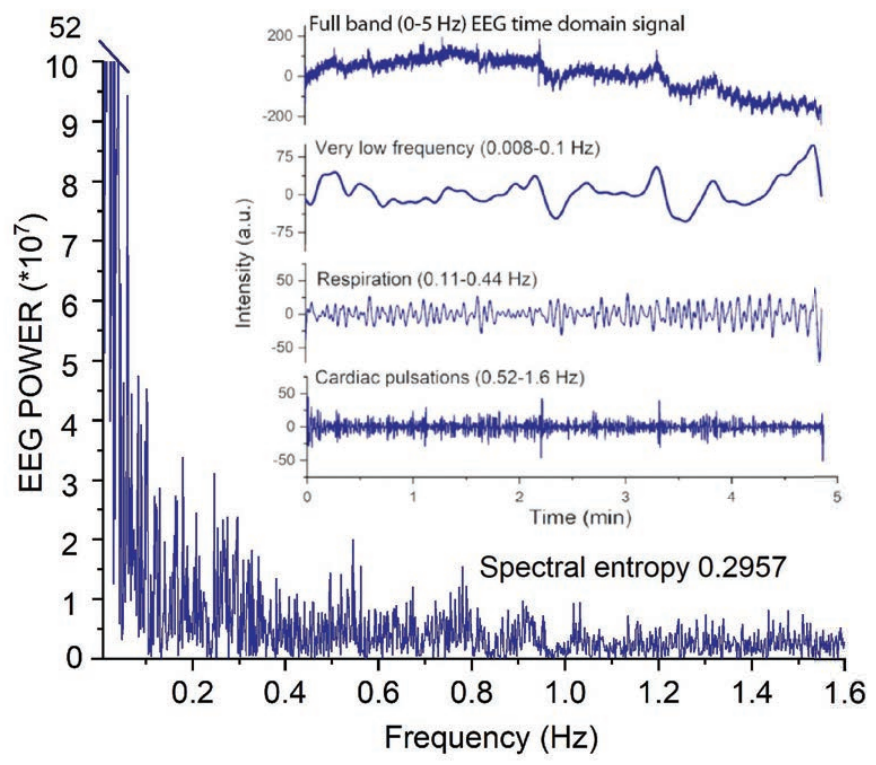

\section{e. Sleep MREG}

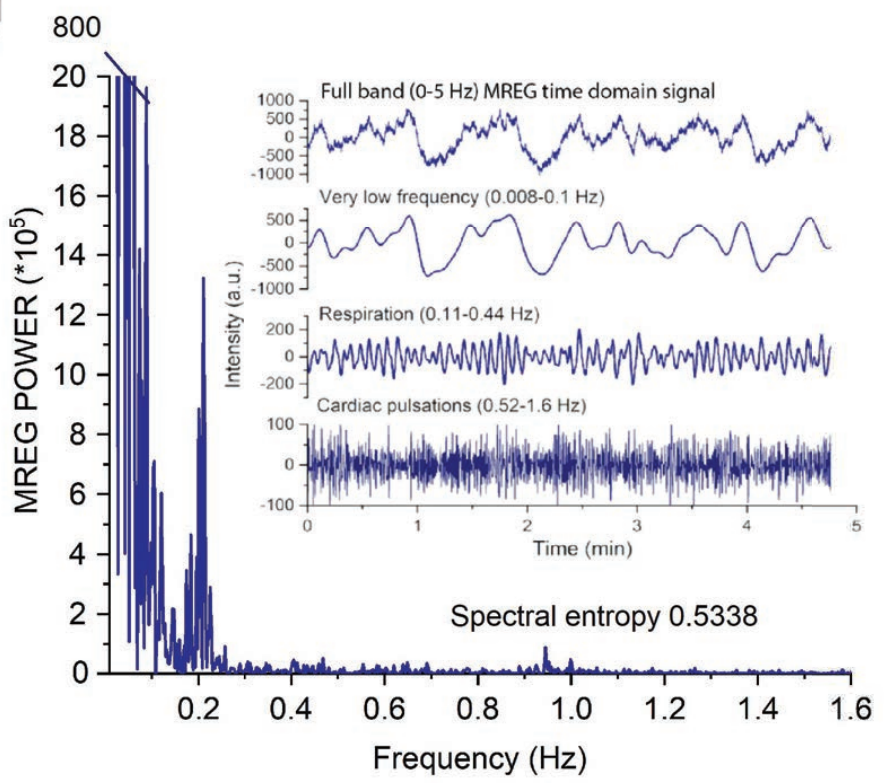


a. EEG sleep state
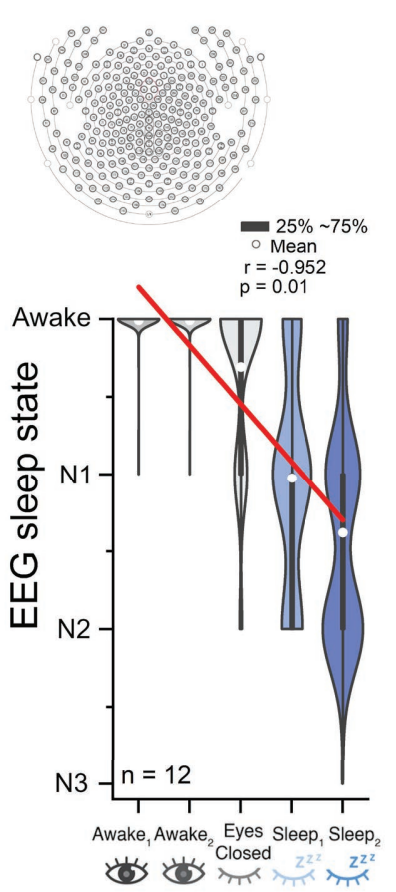

b. Spectral entropy ROI


c. Spectral entropy: Sleep < Awake
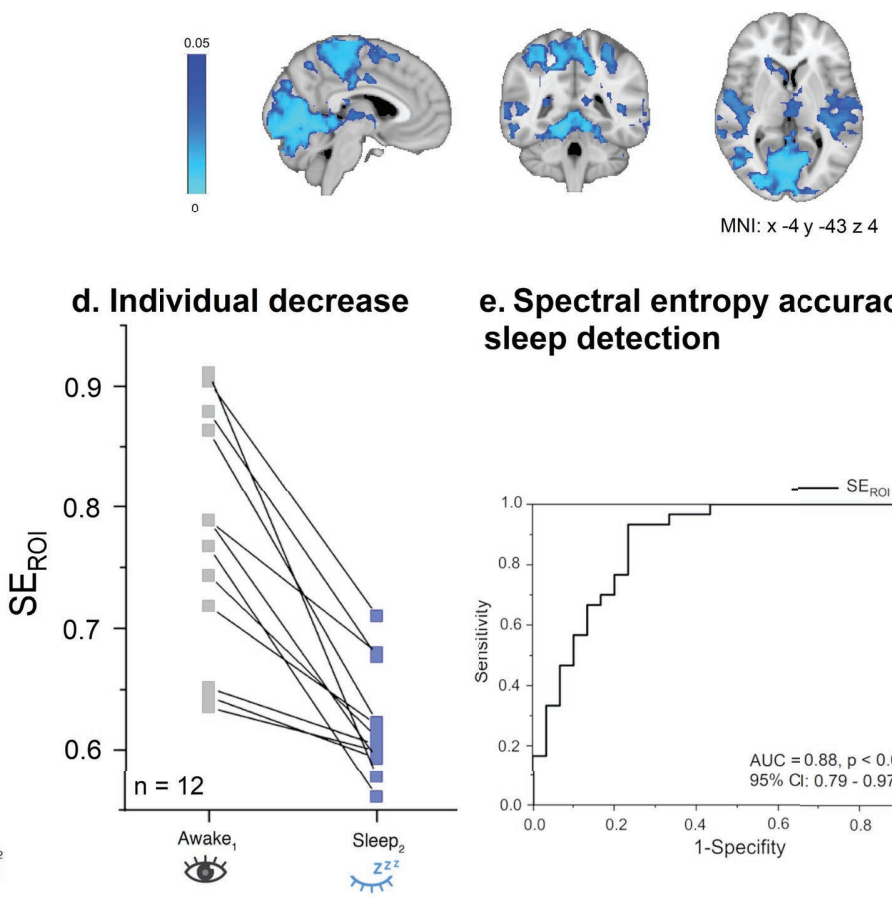

e. Spectral entropy accuracy for sleep detection




a.

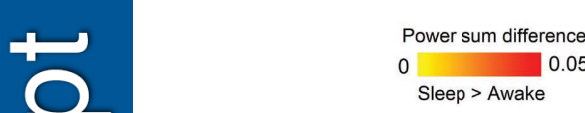

을

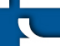

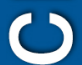

क

3

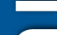

(ิ)

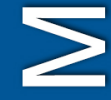

0

(1)

$+$

은

(1)

0

0

4

b.

Sleep > Awake
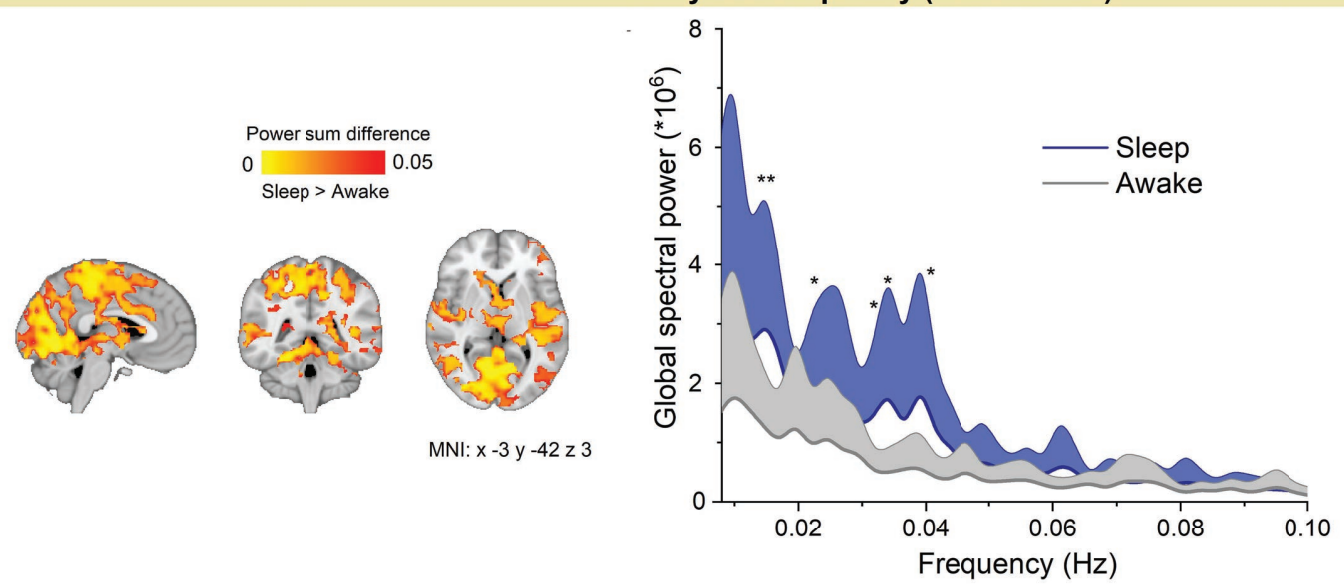

MNI: $x-3$ y $-42 z 3$

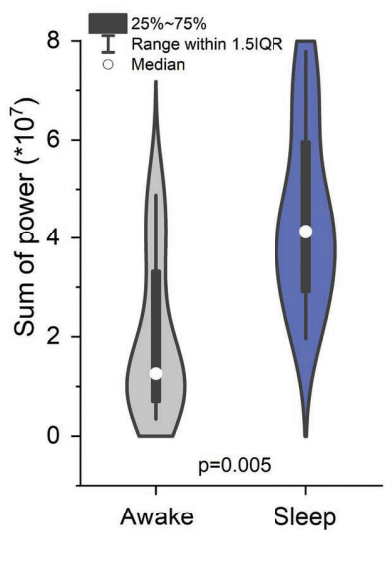

Respiratory frequency $(0.11-0.44 \mathrm{~Hz})$

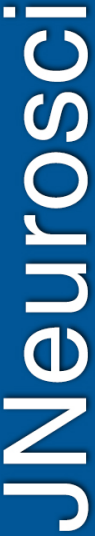

c.

Power sum difference

$0 \quad 0.05$
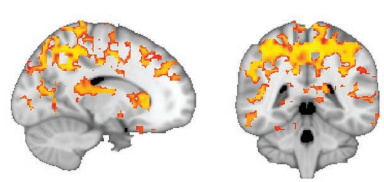

MNI: $x-15$ y -42 z 6
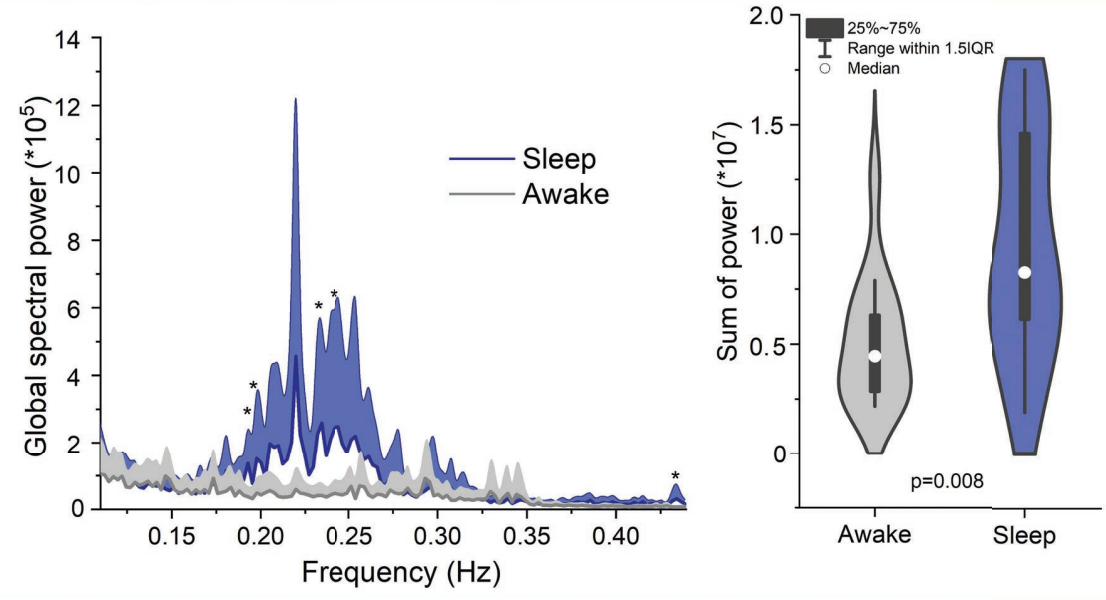

Cardiac frequency $(0.52-1.6 \mathrm{~Hz})$
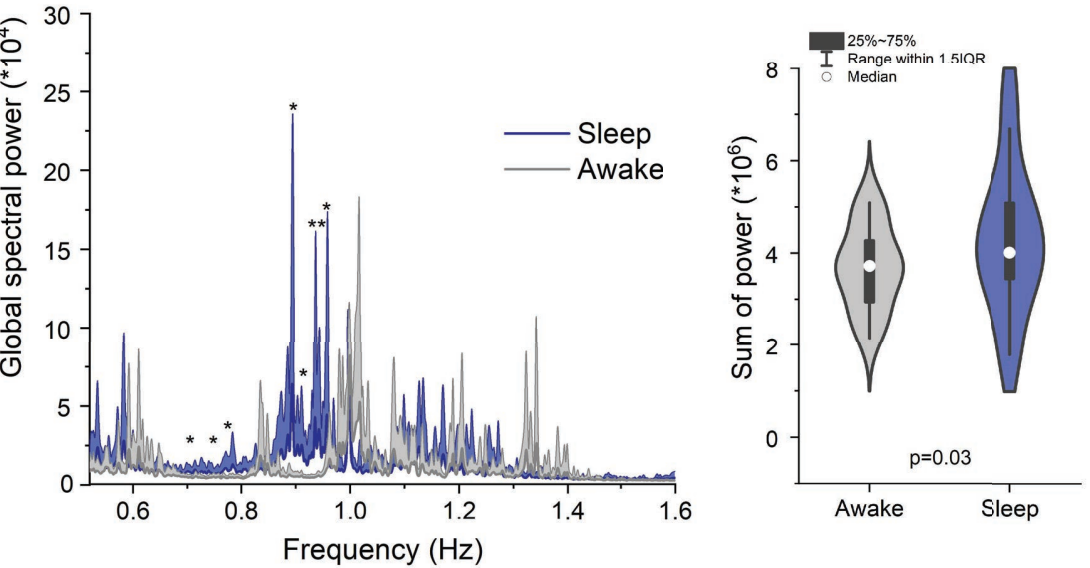


\section{a. Spectral entropy}

(C) ${ }^{0.55}$ Respiratory $(0.11-0.44 \mathrm{~Hz})$



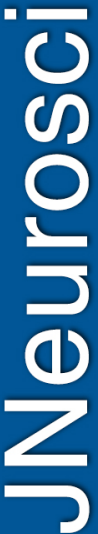

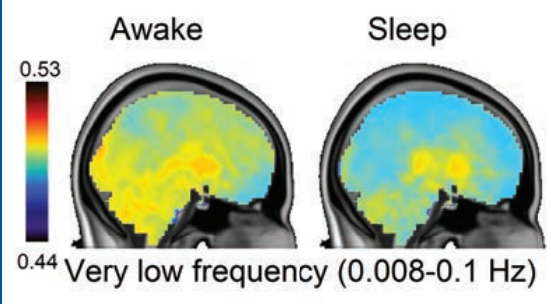



Difference

Sleep vs. Awake
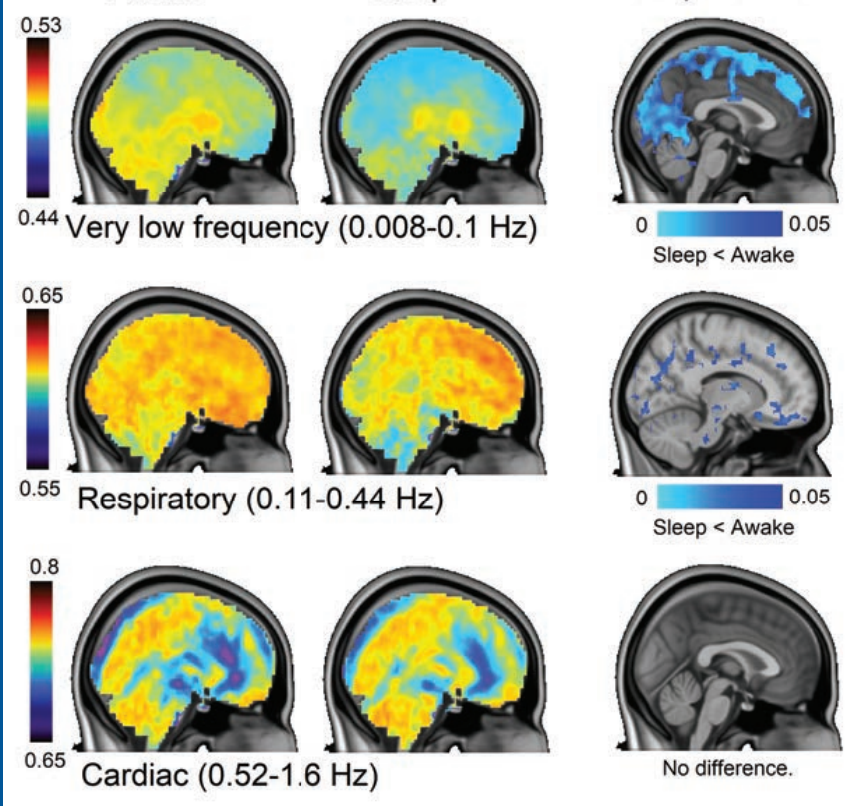

\section{b. Spectral power}

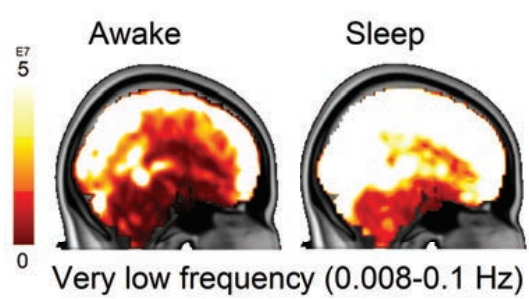

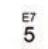



0 Respiratory $(0.11-0.44 \mathrm{~Hz})$

${ }_{5}^{\mathrm{E}}$

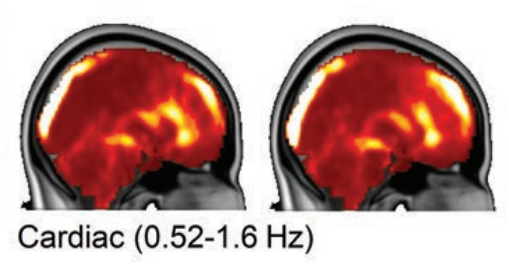

Difference

Sleep vs. Awake
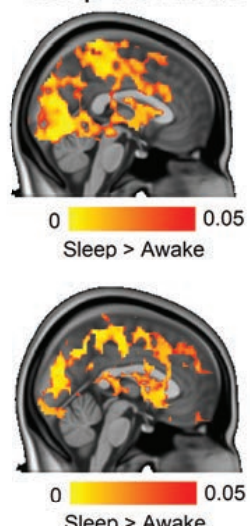

Sleep > Awake






\section{Awake Sleep Sleep $>$ Awake}

a. EEG Slow oscillation $(0.2-2 \mathrm{~Hz})$ power sum
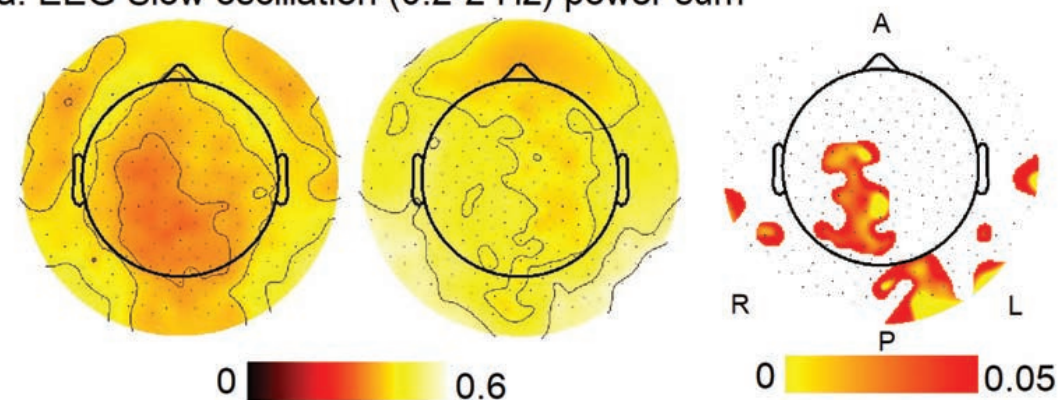

b. MREG: Very low frequency $(0.008-0.1 \mathrm{~Hz})$ power sum


c. MREG: Respiratory frequency $(0.11-0.44 \mathrm{~Hz})$ power sum
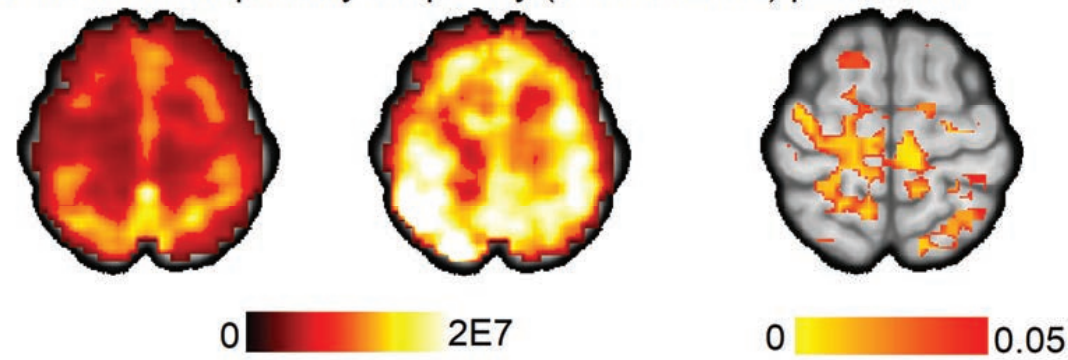

d. MREG: Cardiac frequency $(0.52-1.6 \mathrm{~Hz})$ power sum
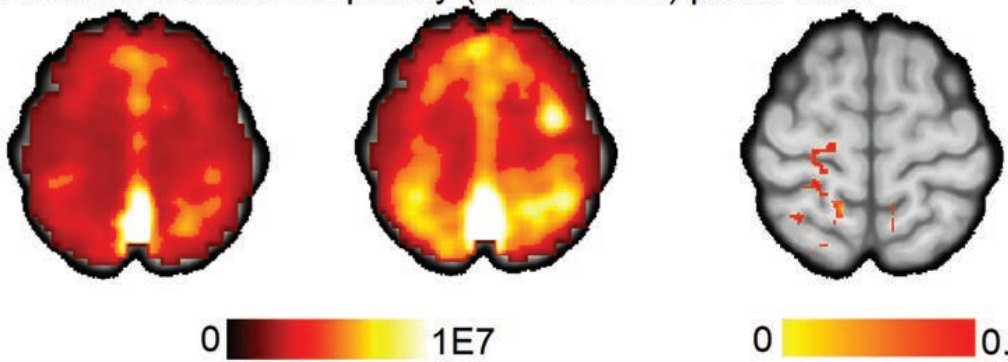

0

0.05

e. MREG: Very low frequency $(0.008-0.1 \mathrm{~Hz})$ spectral entropy

Sleep < Awake
